\title{
The Lift Problem in Flapping Forward Flight at Low Reynolds Numbers
}

CONFERENCE PAPER · JANUARY 2015

DOI: $10.2514 / 6.2015-1750$

4 AUTHORS, INCLUDING:

Tianshu Liu

Western Michigan University

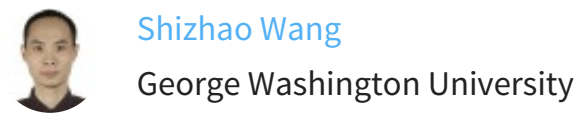

122 PUBLICATIONS 1,146 CITATIONS

26 PUBLICATIONS 95 CITATIONS

SEE PROFILE

SEE PROFILE

Xing Zhang

Chinese Academy of Sciences

51 PUBLICATIONS 257 CITATIONS

SEE PROFILE 


\title{
The Lift Problem in Flapping Forward Flight at Low Reynolds Numbers
}

\author{
Tianshu Liu, ${ }^{1}$ Shizhao Wang ${ }^{2}$, Xing Zhang ${ }^{3}$, Guowei He $^{4}$ \\ Western Michigan University, Kalamazoo, MI 49008, USA \\ The State Key Laboratory of Nonlinear Mechanics, Institute of Mechanics \\ Chinese Academy of Sciences, Beijing 100190, P. R. China
}

\begin{abstract}
This paper discusses the relationship between lift generation and flow structures in flapping forward flight at low Reynolds numbers. The simple lift formula for a wing/body in a sufficiently large rectangular control surface is given in the framework of the general viscous flow theory, which contains the two dominant terms: the vortex lift and the lift associated with the fluid acceleration. This lift decomposition allows quantitative estimation of the contributions of identified flow structures to lift generation. The Kutta-Joukowski theorem and the classical unsteady airfoil theory are the naturally reduced cases from the simple lift formula. The validation and applications of the simple lift decomposition are presented for the flows over the flapping flat-plate airfoil, flapping rectangular morphing wing and flapping bat wing.
\end{abstract}

\section{Introduction}

Low-Reynolds-number flapping flight has recently attracted considerable attention in the aeronautical communities due to the need of developing biologically-inspired micro air vehicles (MAVs) (Plazer et al. 2008, Shyy et al. 2008). Therefore, animal flight, which has been traditionally studied by avian zoologists, becomes immediately relevant to the engineering research. Natural flyers include birds, insects and bats. In bird flight, the Reynolds numbers based on the mean wing chord range from $10^{4}$ to $10^{6}$ while the Reynolds numbers for bat flight are $10^{3}-10^{5}$. In contrast, the Reynolds numbers for insects are typically less than 5000. The complex low-Reynolds-number unsteady aerodynamics in flapping flight poses challenges to researchers in aerodynamics and fluid mechanics because the knowledge and database of the design of fixed-wing aircraft are not suitable in the design of such MAVs. Therefore, it is desirable to understand the physical mechanisms of lift generation in animal flight.

\footnotetext{
${ }^{1}$ Professor, The corresponding author, Department of Mechanical and Aerospace Engineering, Western Michigan University, Kalamazoo, MI 49008, USA, Senior Member, AIAA tianshu.liu@wmich.edu, 269-276-3426

${ }^{2}$ Assistant Professor, The State Key Laboratory of Nonlinear Mechanics, Institute of Mechanics, Chinese Academy of Sciences, Beijing 100190, P. R. China

${ }^{3}$ Associate Professor, The State Key Laboratory of Nonlinear Mechanics, Institute of Mechanics, Chinese Academy of Sciences, Beijing 100190, P. R. China

${ }^{4}$ Professor, The State Key Laboratory of Nonlinear Mechanics, Institute of Mechanics, Chinese Academy of Sciences, Beijing 100190, P. R. China

Copyright $@ 2015$ by the American Institute of Aeronautics and Astronautics, Inc. All rights reserved.
} 
The previous studies have revealed highly complex unsteady flows over flapping wings. To understand the physical mechanisms of flapping flight, it is required to estimate the aerodynamic lift from unsteady velocity fields obtained by computational fluid dynamics (CFD) codes and particle image velocimetry (PIV) measurements in wind tunnels. The connection between lift generation and flow structures is a key to understand flapping flight. The Kutta-Joukowski theorem (the K-J theorem) has been widely used to infer the lift after the circulation is estimated by integrating the vorticity field in a selected cross-section region near wings or in wakes generated in flapping flight. Clearly, the distinct advantage of using the K-J theorem is its simplicity, which is directly related to vorticty fields that can be measured by PIV. In particularly, it allows estimation of the lift components contributed by certain distinct vortical structures such as the leading-edge vortices. On the other hand, to calculate the lift, the K-J theorem has served as an essential mechanism in vortex-based aerodynamics models for low-Reynolds-number flapping flight (Rayner 1979a, 1979b, Ellington 1984, Watts et al. 2001, Hedrick et al. 2002, Spedding et al. 2003). The classical aerodynamics models have been adapted further by incorporating some relevant flow phenomena like the leading- and trailing-edge vortices for additional lift generation (Sane 2003, Ansari et al. 2006). However, the K-J theorem is not accurate to captures the main lift-generating mechanisms in highly unsteady separated flows at low Reynolds numbers in flapping flight. It is necessary to quantitatively examine the lift problem from a perspective of the general viscous flow theory for complex unsteady flows associated with flapping flight.

This paper discusses the relationship between lift generation and flow structures in highly unsteady and separated flows in flapping forward flight at low Reynolds numbers. This paper is organized as follows. The general force expressions and the difficulties in their applications in unsteady separated low-Reynolds number flows are first discussed. Then, the simple lift decomposition into the vortex lift and the lift associated with the fluid acceleration is given, and the Kutta-Joukowski theorem and the unsteady thin-airfoil theory (including the classical Kárman-Sears lift theory) are revisited as the reduced cases. The applications of the simple lift formula are presented for the complex flows over the flapping flat-plate airfoil, flapping rectangular morphing wing and flapping bat wing.

\section{Lift Decompositions}

\subsection{General force expressions}

The force acting on a body immersed in a fluid flow is usually calculated by

$$
\begin{aligned}
\boldsymbol{F} & =-\oint_{\partial B}(-p \boldsymbol{I}+\boldsymbol{\tau}) \cdot \boldsymbol{n} d S \\
& =-\rho \int_{V_{f}(t)} \frac{D \boldsymbol{u}}{D t} d V+\oint_{\Sigma}(-p \boldsymbol{I}+\boldsymbol{\tau}) \cdot \boldsymbol{n} d S,
\end{aligned}
$$

where $p$ and $\tau$ are the pressure and viscous stress tensor, respectively, $\boldsymbol{I}$ is the unit tensor, $V_{f}$ denotes the control volume of fluid, $\partial V_{f}=\partial B+\Sigma$ is the total control surface, $\partial B$ denotes the control surface enclosing the body $B, \Sigma$ denotes an outer control surface in 
which the body is enclosed, and $\boldsymbol{n}$ is the unit normal vector pointing to the outside of a control surface. By using the Navier-Stokes (NS) equations and Gauss's theorem, Eq. (1) can be expressed as

$$
\begin{aligned}
\boldsymbol{F}= & \underbrace{-\rho \int_{V_{f}} \frac{\partial \boldsymbol{u}}{\partial t} d V}_{A}+\underbrace{\rho \int_{V_{f}} \boldsymbol{u} \times \boldsymbol{\omega} d V}_{B}-\underbrace{\oint_{\Sigma} p \boldsymbol{n} d S}_{C} \\
& \underbrace{\oint_{\Sigma} \rho \frac{q^{2}}{2} \boldsymbol{n} d S}_{D}+\underbrace{\oint_{\Sigma} \boldsymbol{n} \cdot \boldsymbol{\tau} d S}_{E}-\underbrace{\oint_{\partial B} \rho \frac{q^{2}}{2} \boldsymbol{n} d S}_{F}
\end{aligned}
$$

where $\boldsymbol{u}$ is the velocity, $\boldsymbol{\omega}$ is the vorticity, and $q=|\boldsymbol{u}|$ is the magnitude of velocity. The term A in Eq. (2) is a volume integral of the local acceleration of fluid induced by a moving solid body and unsteady flow structures as the unsteady inertial effect. The term B is a volume integral of the Lamb vector $\boldsymbol{l}=\boldsymbol{u} \times \boldsymbol{\omega}$, which represents the vortex force. The terms $\mathrm{C}$ and $\mathrm{D}$ are the surface integral of the total pressure (the total head) $p+\rho q^{2} / 2$ on the outer control surface $\Sigma$ and the term $\mathrm{E}$ is the surface shear stress on the outer control surface $\Sigma$. The term $\mathrm{F}$ is the boundary term related to the motion of body boundary $\partial B$. In an inviscid irrotational unsteady flow where the terms B, C, D and E in Eq. (2) vanish, the remaining terms $\mathrm{A}$ and $\mathrm{F}$ together are interpreted as the added-mass force in ideal fluid mechanics. For a thin wing the term $\mathrm{F}$ is small, which is interpreted as the part of the added mass force associated with the fluid virtually occupying the body domain $B$ (a virtual fluid body).

The general force expressions have been extensively discussed by Saffman (1992) in the inviscid-flow framework. From the NS equations, various force expressions have been derived depending on how to transform the troublesome pressure term to the velocity-related quantities. Noca et al. (1999) gave the force expressions called 'Impulse Equation', 'Momentum Equation' and 'Flux Equation'. Wu et al. $(2005,2006)$ derived the force expressions by using "derivative-moment transformations". By introducing an auxiliary velocity potential satisfying suitable boundary conditions and projecting the NS equations on the gradient of the potential, Quartapelle \& Napolitano (1983), Howe (1989), Chang (1992) and Lee et al. (2012) were able to extract the explicit pressure force from the pressure term and decompose the force into several terms whose physical meanings become clearer. In principle, a general lift formula can be derived by directly projecting these general force expressions onto the direction normal to the incoming flow. Many terms appear as a consequence of eliminating the pressure term, and thus a concise form of the lift expressions cannot be directly obtained in such a way. Furthermore, the physical meanings of some terms in these lift expressions cannot be easily elucidated and their relative contributions to the lift cannot be clearly distinguished. Although various lift decompositions into the elemental terms are equivalent, from a standpoint of application, good lift decomposition should have a minimal number of the terms with lucid physical meanings such that the flow structures responsible to lift generation can be clearly identified. 


\subsection{Simple lift formula}

It is recently recognized that the troublesome pressure term can be neglected in the first-order approximation when a sufficiently large rectangular control domain is considered (Wang et al. 2013). The lift on a body is given by $F_{z}=\boldsymbol{k} \cdot \boldsymbol{F}$, where $\boldsymbol{k}$ is the unit vector normal to the freestream. A rectangular domain $D$ is selected as a control volume to simplify the lift expression (as shown in Fig. 2). For a sufficiently large boundary $\Sigma$, the viscous stress term $\boldsymbol{k} \cdot(\boldsymbol{\tau} \cdot \boldsymbol{n})$ on the most portion of $\Sigma$ is zero except in a wake, and thus the contribution of the term $\mathrm{E}$ to the lift can be neglected. The major problem is to estimate the term $\mathrm{C}+\mathrm{D}$ in the RHS of Eq. (2) that is the surface integral of the total pressure $p+\rho q^{2} / 2$ on a fixed rectangular outer control surface $\Sigma$. The total pressure is $p+\rho q^{2} / 2=C(t)-\rho \partial \phi / \partial t$, where $\phi$ is the velocity potential and $C(t)$ is an unknown function. Since the unit normal vector $\boldsymbol{n}$ on the four vertical faces of the rectangular surface is perpendicular to $\boldsymbol{k}(\boldsymbol{k} \cdot \boldsymbol{n}=0)$, the integral in $\mathrm{C}+\mathrm{D}$ on the four vertical faces is zero. The remaining part of $\mathrm{C}+\mathrm{D}$ is contributed by the integral on the top and bottom faces of the rectangular outer control surface, which is proportional to $\left\langle\phi^{+}\right\rangle_{A}-\left\langle\phi^{-}\right\rangle_{A}$ and $\left\langle C^{+}\right\rangle_{A}-\left\langle C^{-}\right\rangle_{A}$, where \langle\rangle$_{A}$ is the area-averaging operator and the superscripts + and denote the values at the top and bottom faces. When on the top and bottom faces moves sufficiently away from a wing, the value of $\left\langle\phi^{+}\right\rangle_{A}-\left\langle\phi^{-}\right\rangle_{A}$ approaches to zero since $\phi \sim|z|^{-n} \rightarrow 0$ as $|z| \rightarrow \infty$, where $|z|$ is the distance of the top or bottom face to the wing, and $n$ is a positive exponent to be determined. The difference $\left\langle C^{+}\right\rangle_{A}-\left\langle C^{-}\right\rangle_{A}$ represents the asymmetry of the far-field flow with respect to the $x$-axis. When the freestream flow is steady, $C(t)$ is constant since $\phi \sim r^{-n} \rightarrow 0$ as $r \rightarrow \infty$, and therefore $\left\langle C^{+}\right\rangle_{A}-\left\langle C^{-}\right\rangle_{A}=0$. In normal forward flapping flight, therefore, it is plausible to assume that the total pressure term is small for a sufficiently large rectangular domain. However, the above analysis is qualitative. The values of the total pressure term and the terms $\mathrm{E}$ and $\mathrm{F}$ in a finite domain have been quantitatively evaluated through direct numerical simulations (DNS) for a flapping flat plate and a flapping rectangular wing (Wang et al 2013, 2014a, 2014b).

Under the above approximations, the simple lift formula for forward flight is given in the two dominant terms, i.e.,

$$
L \approx L_{v o r}+L_{a c c}
$$

where the vortex lift (the Lamb vector integral) is

$$
L_{v o r}=\rho \boldsymbol{k} \cdot \int_{V_{f}} \boldsymbol{u} \times \boldsymbol{\omega} d V,
$$


and the lift associated with the fluid acceleration is

$$
L_{a c c}=-\rho \boldsymbol{k} \cdot \int_{V_{f}} \frac{\partial \boldsymbol{u}}{\partial t} d V-\rho \boldsymbol{k} \cdot \oint_{\partial B}\left(|\boldsymbol{u}|^{2} / 2\right) \boldsymbol{n} d S .
$$

Furthermore, Eq. (5) can be written as

$$
L_{a}=-\rho \boldsymbol{k} \cdot \frac{d}{d t} \int_{V_{f}} \boldsymbol{u} d V+\rho \boldsymbol{k} \cdot \oint_{\Sigma} \boldsymbol{n} \cdot\left[\frac{1}{2}|\boldsymbol{u}|^{2} \boldsymbol{I}-\boldsymbol{u} \boldsymbol{u}\right] d S=-\rho \boldsymbol{k} \cdot \frac{d}{d t} \int_{V_{f}} \boldsymbol{u} d V,
$$

since $\boldsymbol{k} \cdot \boldsymbol{n}=0$ on the vertical faces and $\boldsymbol{k} \cdot \boldsymbol{u} \rightarrow \boldsymbol{k} \cdot \boldsymbol{i} U=O$ on the top and bottom faces, the term $\boldsymbol{k} \cdot \boldsymbol{n} \cdot\left(0.5|\boldsymbol{u}|^{2} \boldsymbol{I}-\boldsymbol{u u}\right)$ approaches to zero. Due to the formal simplicity and physical clarity of Eq. (3), it is particularly useful to evaluate the contributions of distinct vortical structures to the lift in unsteady flows. In a limiting case where a moving body is in a completely inviscid irrotational flow with $\boldsymbol{u}=\nabla \phi, L_{a}$ is interpreted as the added-mass force projected on the direction of $\boldsymbol{k}$. In this case, $L_{a}$ is expressed as $L_{a}=m_{a} d U_{b} / d t$, where the added mass is defined as $m_{a} \approx-\rho l_{r e f} \int_{\partial B} \bar{\phi}(\boldsymbol{k} \cdot \boldsymbol{n}) d S, \bar{\phi}=\phi\left[U_{b}(t) l_{r e f}\right]^{-1}$ is the normalized velocity potential, and $U_{b}(t)$ and $l_{r e f}$ the velocity and the reference length of the body, respectively.

From a theoretical standpoint, the selection of a particular control surface imposes a constraint on the generality of the simple lift formula, but it is convenient in applications since a rectangular domain is usually used anyway in processing of computational and experimental data. In addition, the form of the Lamb vector integral and the local vertical acceleration allows domain decomposition in which they can be expressed as a sum of the corresponding terms in sub-domains. This is advantageous since the contributions of individual flow structures in sub-domains to the lift can be directly distinguished and evaluated.

\subsection{The Kutta-Joukowski theorem}

In two dimensions (2D), the sectional lift given by Eq. (3) is

$$
L^{\prime}=\rho D\left(\left\langle u_{x} \omega_{y}\right\rangle_{D}-\left\langle\partial u_{z} / \partial t\right\rangle_{D}\right)+\rho V_{B}\langle q \partial q / \partial z\rangle_{B},
$$

where $\boldsymbol{u}=\left(u_{x}, u_{z}\right)$ is the fluid velocity vector, the $x$ - and $z$-coordinates are in the freestream and vertical directions, respectively, $D$ denotes the domain area in two dimensions, and $\langle\bullet\rangle_{D}$ becomes the area-averaged operator in 2D. To compare Eq. (7) with the K-J theorem, the local effective velocity (the vorticity-weighted velocity) is defined as $u_{\text {eff }}=u_{x} \omega_{y} /\left\langle\omega_{y}\right\rangle_{D}$, where $\left\langle\omega_{y}\right\rangle_{D}$ is the area-averaged vorticity. Further, by introducing 
the area-averaged effective velocity $U_{\text {eff }}=\left\langle u_{\text {eff }}\right\rangle_{D}$, Eq. (7) is written in a form analogous to the K-J theorem, i.e.,

$$
L^{\prime}=\rho U_{e f f} \Gamma-\rho D\left\langle\partial u_{z} / \partial t\right\rangle_{D}+\rho V_{B}\langle q \partial q / \partial z\rangle_{B}
$$

where $\Gamma=\left\langle\omega_{y}\right\rangle_{D} D$ is the circulation. Unlike the classical K-J theorem $L_{K-J}^{\prime}=\rho U_{\infty} \Gamma$, the domain-averaged effective velocity $U_{\text {eff }}$ rather than $U_{\infty}$ is used in Eq. (8), which depends on not only the velocity and vorticity distributions around a body but also time in general. For unsteady flows, application of the K-J theorem implies the quasi-steady-state assumption in which $L_{K-J}^{\prime}(t)$ is synchronized with $\Gamma(t)$. This assumption is generally problematic, which could lead to errors in the time-averaged magnitude and phase of the unsteady lift. To illustrate this point, by introducing a velocity difference $v(t)=U_{e f f}(t)-U_{\infty}$ that represents a certain perturbation velocity, Eq. (8) gives $L^{\prime}(t)-L_{K-J}^{\prime}(t)=\rho v(t) \Gamma(t)-\rho D\left\langle\partial u_{z} / \partial t\right\rangle_{D}+\rho V_{B}\langle q \partial q / \partial z\rangle_{B}$. Clearly, it is indicated that $L^{\prime}(t)$ and $L_{K-J}^{\prime}(t)$ could considerably differ in the phase, amplitude and waveform of the unsteady lift particularly when the velocity fluctuation $v(t)$ and the acceleration term are large. The corresponding relation for the time-averaged lift is $\overline{L^{\prime}}-{\overline{L^{\prime}}}_{K-J}=\rho \overline{v \Gamma}-\rho D\left\langle\overline{\partial u_{z} / \partial t}\right\rangle_{D}+\rho V_{B}\langle\overline{q \partial q / \partial z}\rangle_{B}$, where the bar - denotes the time-average.

The classical K-J theorem is a reduced form of Eq. (8). In a steady $2 \mathrm{D}$ flow where $\left\langle\partial u_{z} / \partial t\right\rangle_{D}=0$ and $V_{B}\langle q \partial q / \partial z\rangle_{B}=O$, a vorticity patch bounded in a finite region that is much smaller than the rectangular control domain $D$ is considered. The $x$-component velocity can be decomposed into $u_{x}=U_{\infty}+u_{x}^{\prime}$, where $u_{x}^{\prime}$ is the velocity induced by the vorticity patch along the $x$-coordinate that is given by

$$
u_{x}^{\prime}(x, z)=\frac{1}{2 \pi} \iint_{D} \frac{(z-\eta) \omega_{y}(\xi, \eta)}{(x-\xi)^{2}+(z-\eta)^{2}} d \xi d \eta
$$

Therefore, since $\quad u_{\text {eff }}=u_{x} \omega_{y} /\left\langle\omega_{y}\right\rangle_{D}=U_{\infty} \omega_{y} /\left\langle\omega_{y}\right\rangle_{D}+u_{x}^{\prime} \omega_{y} /\left\langle\omega_{y}\right\rangle_{D}, \quad$ the domain-averaged effective velocity is

$$
U_{e f f}=\left\langle u_{e f f}\right\rangle_{D}=U_{\infty}+\frac{1}{2 \pi} \frac{1}{\left\langle\omega_{y}\right\rangle_{D} D} \iiint \int \frac{(z-\eta) \omega_{y}(\xi, \eta) \omega_{y}(x, z)}{(x-\xi)^{2}+(z-\eta)^{2}} d \xi d \eta d x d z
$$

where the integration domain in both $(\xi, \eta)$ and $(x, z)$ is $D$. When the variables $(\xi, \eta)$ 
and $(x, z)$ are interchanged, the factor $z-\eta$ in Eq. (7) changes its sign (Kárman and Burgers 1963). It is known that the integral in Eq. (10) must vanish such that $U_{\text {eff }}=U_{\infty}$. In this case, the K-J theorem $L_{K-J}^{\prime}(t)=\rho U_{\infty} \Gamma$ is exactly recovered from Eq. (8).

\subsection{Unsteady thin-airfoil theory}

Based on the simple lift formula Eq. (3), the physical foundations of the unsteady thin airfoil theory are explored in the viscous-flow framework (Liu et al. 2014). A 2D attached flow field over a thin airfoil can be decomposed into the outer potential flow and the boundary layer (or the viscous flow region). Therefore the velocity is expressed as $\boldsymbol{u}=\nabla \phi H\left(\boldsymbol{x} \in D_{\text {out }}\right)+\boldsymbol{u} H\left(\boldsymbol{x} \in D_{b l}\right)$, where $\phi$ is the velocity potential, $D_{\text {out }}$ and $D_{b l}$ denote the outer flow domain and the boundary layer domain $\left(V_{f}=D_{o u t}+D_{b l}\right)$, respectively, and the Heaviside function is defined as $H(x \in D)=1$ and $H(x \notin D)=0$. The boundary-layer edge is denoted by $\partial B_{b l}$ that separates the two domains. In this case, the vortex lift is solely contributed by the boundary layer. For a 2D attached flow, according to Eq. (8), the lift per unit span is given by

$$
L^{\prime}=L_{v o r}^{\prime}+L_{a}^{\prime} \approx \rho U_{e f f} \Gamma+\rho \frac{d}{d t} \int_{\partial B_{b l}} \phi\left(\boldsymbol{k} \cdot \boldsymbol{n}^{\prime}\right) d S,
$$

where $\Gamma=\left\langle\omega_{y}\right\rangle_{D} D$ is the circulation, $U_{\text {eff }}=\left\langle u_{\text {eff }}\right\rangle_{D}$ is the area-averaged effective velocity, $u_{e f f}=u \omega_{y} /\left\langle\omega_{y}\right\rangle_{D},\left\langle\omega_{y}\right\rangle_{D}$ is the area-averaged spanwise vorticity, $u$ is the velocity component in the $x$-direction, and $n^{\prime}$ is the unit normal vector pointing outward from the wing surface $\left(\boldsymbol{n}^{\prime}=-\boldsymbol{n}\right)$. Here, $L_{a}^{\prime}$ in Eq. (11) mainly represents the added-mass lift associated with the unsteady outer flow induced by a moving wing.

Further, for a thin airfoil, the circulation is given by the integral of the physical quantity $\gamma(x, t)$ along the coordinate $x$ on the chord line of the airfoil, i.e.,

$$
\Gamma=\int_{D_{b l}} \omega_{y} d S=\int_{x_{L E}}^{x_{T E}} \gamma(x, t) d x .
$$

In Eq. (12), $\gamma(x, t)$ is defined as

$$
\gamma(x, t)=\int_{0}^{\delta^{+}} \omega_{y}^{+} d n^{++}+\int_{0}^{\delta^{-}} \omega_{y}^{-} d n^{\prime-} \approx u_{x e}^{+}-u_{x e}^{-}
$$


where the superscripts + and - denote the quantities on the upper and lower surfaces of the thin airfoil, respectively, $\delta$ denotes the boundary-layer thickness, $n^{\prime}$ is the normal coordinate directing outward from the thin airfoil surface, and $u_{x e}^{+}-u_{x e}^{-}$is the velocity difference between the boundary-layer edges on the upper and lower surfaces. The function $\gamma(x, t)$ is a lumped model of the vorticity distribution on the airfoil surface. In the limiting case where the boundary layer becomes very thin as the Reynolds number is increased, $\gamma(x, t)$ is interpreted as the strength of a vortex sheet in the classical thin airfoil theory.

The added-mass lift per unit span is

$$
\begin{aligned}
L_{a}^{\prime} & \approx \rho \frac{d}{d t} \int_{\partial B_{b l}} \phi\left(\boldsymbol{k} \cdot \boldsymbol{n}^{\prime}\right) d S \approx \rho \frac{d}{d t} \int_{x_{L E}}^{x_{T E}}\left(\phi_{e}^{+}-\phi_{e}^{-}\right) d x \\
& =\rho \frac{d}{d t} \int_{x_{L E}}^{x_{T E}}\left(x_{r e f}-x\right) \gamma(x, t) d x
\end{aligned}
$$

where $x_{L E}$ and $x_{T E}$ are the leading-edge and trailing-edge locations, respectively, and the subscript ' $\mathrm{e}$ ' denotes the boundary-layer edge. In the derivation of the second approximate equality in Eq. (14), it is assumed that $\left(\boldsymbol{k} \cdot \boldsymbol{n}^{\prime}\right) d S \approx d x$ in 2D. Another approximation is that the effect of the unsteady boundary layer edge $\partial B_{b l}$ is neglected since the edge locations $x_{L E}$ and $x_{T E}$ are treated as the time-independent variables. In the derivation of the last equality in Eq. (14), integration by parts is carried out and the reference location $x_{r e f}$ is introduced as a parameter when the mean value theorem is applied. The reference location $x_{r e f}$ is determined in the classical flow across an accelerating flat plate in which $\bar{x}_{\text {ref }}=0.5$ is found (Liu et al. 2014). It is interesting that $L_{a}^{\prime}$ in Eq. (9) is reduced to the time rate of the vortex impulse (or vortex moment) in the unsteady thin airfoil theory.

Substitution of Eqs. (12) and (14) into Eq. (11) yields the thin-airfoil lift formula (TALF)

$$
L^{\prime}(t)=L_{\text {vor }}^{\prime}(t)+L_{a}^{\prime}(t) \approx \rho U(t) c \int_{0}^{l} \gamma(\bar{x}, t) d \bar{x}+\rho c^{2} \frac{d}{d t} \int_{0}^{l}\left(\bar{x}_{r e f}-\bar{x}\right) \gamma(\bar{x}, t) d \bar{x},
$$

where $\bar{x}=\left(x-x_{L E}\right) / c$ is the normalized coordinate, $\bar{x}_{r e f}=\left(x_{r e f}-x_{L E}\right) / c$ is the normalized reference location, and $c$ is the chord, The first and second terms in the RHS of in Eq. (15) are interpreted as the Kutta-Joukowski lift and the added-mass lift, respectively. The above analysis shows how Eq. (11) is mathematically reduced to Eq. (15) in which a 
vortex sheet is considered as an idealized model of a boundary layer (or near-wall shear layer) for a thin airfoil. The above analysis provides a systematical and quantitative support to the arguments by Glauert (1983) and Sears (1976) on the physical foundations of the thin airfoil theory in which a vortex sheet is a limiting model of a boundary layer on the airfoil surface as the viscosity approaches to zero. Sears (1976) obtained Eq. (15) using the unsteady Bernoulli equation in the inviscid-flow framework, but argued physically that it could be correct even when the boundary layer is separated. In fact, the aforementioned derivation of Eq. (15) can be extended to moderately separated flows by defining the limits $\delta^{+}$and $\delta^{-}$ in the integral of the vorticity field [Eq. (13)] as the sufficiently large distances from the airfoil surface beyond the viscous flow region. In this sense, the whole separated flow plus an airfoil is vertically compressed into a vortex sheet with the strength $\gamma(x, t)$. When $\gamma(x, t)$ is calculated from the vorticity fields obtained from global velocity measurements and CFD, the nonlinear effects associated with viscous separated flows could be naturally incorporated in Eq. (15) particularly in the vortex lift.

From a standpoint of theoretical aerodynamics, to formally isolate the effect of the wake, a decomposition $\gamma=\gamma_{0}+\gamma_{1}$ is used, where $\gamma_{0}$ is the quasi-steady part without considering the effect of the wake vortex sheet and $\gamma_{1}$ is the unsteady part induced by the wake. The analytical solution of the thin-airfoil equation gives the relation between $\gamma_{1}$ and the wake strength $\gamma_{w}$

$$
\gamma_{1}(\bar{x}, t)=\int_{1}^{\infty} G\left(\bar{\xi}_{w} ; \bar{x}, t\right) \gamma_{w}\left(\bar{\xi}_{w}, t\right) d \bar{\xi}_{w},
$$

where the Green's function is

$$
G\left(\bar{\xi}_{w} ; \bar{x}, t\right)=\frac{2}{\pi \sqrt{\bar{x}(1-\bar{x})}} \int_{0}^{1} E(\bar{\xi}, \bar{x}) S\left(\bar{\xi}_{w} ; \bar{\xi}, t\right) d \bar{\xi},
$$

and

$$
E(\bar{\xi}, \bar{x})=\frac{\sqrt{\bar{\xi}(1-\bar{\xi})}}{\bar{x}-\bar{\xi}}-\frac{\sqrt{\bar{\xi}(1-\bar{\xi})}}{1-\bar{\xi}}
$$

Substituting $\gamma=\gamma_{0}+\gamma_{1}$ into Eq. (15) and using Eq. (16) and an integral formula given by Kárman and Sears (1938) [Eq. (15) in their paper] for the wake vortices traveling with the freestream velocity $U$, we have 


$$
\begin{aligned}
L^{\prime}(t) \approx & \rho U(t) c \int_{0}^{l} \gamma_{0}(\bar{x}, t) d \bar{x}+\rho c^{2} \frac{d}{d t} \int_{0}^{l}\left(\bar{x}_{r e f}-\bar{x}\right) \gamma_{0}(\bar{x}, t) d \bar{x} \\
& +\rho U(t) c \int_{1}^{\infty} \gamma_{w}(\bar{\xi}, t) P(\bar{\xi}, t) d \bar{\xi}
\end{aligned}
$$

where the relevant functions in the wake terms are defined as

$$
\begin{aligned}
& P(\bar{\xi}, t)=\frac{d H_{1}(\bar{\xi}, t)}{d \bar{\xi}}+H_{2}(\bar{\xi}, t), \\
& H_{1}(\bar{\xi}, t)=\int_{0}^{1}\left(\bar{x}_{r e f}-\bar{x}\right) G(\bar{\xi} ; \bar{x}, t) d \bar{x}, \\
& H_{2}(\bar{\xi}, t)=\int_{0}^{l} G(\bar{\xi} ; \bar{x}, t) d \bar{x} .
\end{aligned}
$$

In Eq. (19), the first term in the RHS is the quasi-steady vortex lift, the second term is the added-mass lift, and the third term describes the effect of the wake on the lift which explicitly depends on the strength distribution of the wake vortex sheet.

Clearly, Eq. (19) is a reduced form of Eq. (15) when the decomposition $\gamma=\gamma_{0}+\gamma_{1}$ is introduced and the solution of the thin airfoil equation is applied. On the other hand, Eq. (19) is considered as a generalized form of the Kárman-Sears lift formula. When a rigid wake vortex sheet is aligned and traveled with the freestream velocity, Kárman and Sears (1938) gave a specific time-independent Green's function

$$
G(\bar{\xi} ; \bar{x})=\frac{1}{\pi} \frac{1}{\bar{\xi}-\bar{x}} \sqrt{\frac{1-\bar{x}}{\bar{x}}} \sqrt{\frac{\bar{\xi}}{\bar{\xi}-1}} .
$$

Therefore, the Green's function $P(\bar{\xi})$ in the wake-induced term is

$$
P(\bar{\xi})=\frac{0.5-\bar{x}_{r e f}}{4 \sqrt{\bar{\xi}(\bar{\xi}-1)^{3}}}+\frac{1}{2 \sqrt{\bar{\xi}(\bar{\xi}-1)}} .
$$

Since $\bar{x}_{r e f}=0.5$ that is determined by using a particular solution for the classical flow across an accelerating flat plate, Eq. (19) gives the classical Kárman-Sears lift formula

$$
\begin{aligned}
L^{\prime}(t)= & \rho U(t) c \Gamma_{0}+\rho c^{2} \frac{d}{d t} \int_{0}^{1}(0.5-\bar{x}) \gamma_{0}(\bar{x}, t) d \bar{x} \\
& +\rho U(t) c \int_{1}^{\infty} \gamma_{w}(\bar{\xi}, t) \frac{0.5 d \bar{\xi}}{\sqrt{\bar{\xi}(\bar{\xi}-1)}}
\end{aligned}
$$

The unsteady thin airfoil theory is revisited by applying the simple lift formula that is accurate for a sufficiently large rectangular control volume enclosing a wing in viscous flows. 
Eq. (11) allows a direct derivation of the thin-airfoil lift formula Eq. (15) for a 2D flow field decomposed into the outer potential flow and the boundary layer, which contains the Kutta-Joukowski lift and the added-mass lift. This derivation illustrates that the viscous flow around an airfoil is essentially compressed into a vortex sheet with the strength calculated by integrating the vorticity field around the airfoil along the vertical direction. From this perspective, Eq. (15) could be applicable even when the flow around the airfoil is moderately separated. Further, after the vortex sheet strength distribution is decomposed into the quasi-steady part and the wake-induced part, Eq. (15) recovers the classical Kárman-Sears lift formula.

The above analysis of the thin airfoil elucidates a direct link between boundary layer and lift generation since the Lamb vector as the sole constituent of the vortex lift is concentrated near the wall in the boundary layer. The lift generation of a wing is considered as a viscous-flow phenomenon, which means that no lift is generated in an ideal inviscid fluid. The legitimacy of the potential-flow models in classical aerodynamics must be considered in the viscous-flow framework, in which the Kutta condition is imposed to collectively simulate the effects of viscous flows over the airfoil as an empirical condition. The correctness of the Kutta condition for steady attached flows is reasonably validated, but its feasibility for highly unsteady separated flows is questionable. Since a boundary layer is also the source of the form drag (including the skin friction drag), the lift problem is always physically coupled with the drag problem although they could be, to certain degree, mathematically treated separately in approximations. From this perspective, the form drag could be considered as a cost of generating the lift in addition to the induced drag.

\section{Validation and Applications}

\subsection{Flapping flat-plate airfoil}

To evaluate the accuracy of the simple lift formula (SLF) the thin-airfoil lift formula (TALF), the 2D flows over a flapping flat-plate airfoil at a low Reynolds number are investigated through direct numerical simulations (DNS). The plate has a chord length $c$ and zero thickness, heaving vertically and rotating around the center of the plate in a uniform flow. The Cartesian system $O-X Y Z$ fixed in the space is used in DNS. As shown in Fig. 3 , the $X$-axis directs downstream in parallel to the freestream, the $Z$-axis is perpendicular to the freestream pointing upward, the $Y$-axis is normal to the $X Y$ plane along the wingspan. The kinematics of the flapping plate is described by the pitching angle (the geometrical angle of attack, AoA) and the vertical position of the center of the plate, i.e., $\alpha=\alpha_{0}+\alpha_{m} \cos (2 \pi f t)$ and $z_{c}=z_{c 0}+A \sin (2 \pi f t)$, where $\alpha$ is the instantaneous AoA, $\alpha_{0}$ is the time-averaged AoA, and $\alpha_{m}$ is the pitching amplitude. $z_{c}$ is the vertical position of the center of the plate, $z_{c 0}$ is the time-averaged vertical position of the center, and $A$ is the heaving amplitude. $f$ is the pitching and/or heaving frequency. The reduced frequency of the flapping wing is defined as $k=\pi f c / U$. The kinematic parameters in the 
four cases studied in DNS are given in Table 1. The flow around the flapping airfoil is determined by the incompressible NS equations (Wang et al. 2014). The Reynolds number based on the chord length of the plate and uniform upstream flow is $R e=U c / v=300$. The unsteady flows with the moving boundaries are simulated by using the immersed boundary (IB) method based on the discrete stream function formulation developed by Wang and Zhang (2011).

The four cases shown in Table 1 are considered for a flapping flat-plate airfoil at $R e=U c / v=300$. The low-frequency flapping motion is first studied in Case A and Case $\mathrm{C}$ in which the reduced frequency is $k=\pi f c / U=0.06 \pi$. However, in Case $\mathrm{C}$, the non-dimensional heaving amplitude is $A / c=0.25$ and the time-averaged AOA is $\alpha_{0}=10^{\circ}$, which are ten time as large as those in Case A. Figures 4(a) and 5(a) show the vorticity fields around the airfoil at the instants when the lift reaches the maximum and minimum for Case A. Although the boundary layer is weakly separated the sharp leading edge in Case A, the shear layers are still close to the upper and lower surfaces of the airfoil in Case A in which the flapping frequency and heaving amplitude and the time-averaged AOA are small. In contrast, the flow around the airfoil in Case $\mathrm{C}$ is considerably separated since the heaving amplitude and the time-averaged AoA are much larger.

Figure 6(a) shows the histories of the lift coefficient given by the simple lift formula (SLF) and the thin-airfoil lift formula (TALF) for Case A, where the DNS data are obtained by directly integrating the surface pressure and skin friction fields. The SLF is in excellent agreement with the DNS in all the cases. It is expected that the TALF gives the result that is consistent with the DNS data in Case A and the waveform of the lift coefficient remains sinusoidal. More interestingly, as shown in Fig. 6(c), the TALF gives a good prediction of the lift coefficient in Case $\mathrm{C}$ where the flow around the airfoil is considerably separated. The waveform of the lift coefficient is no longer sinusoidal, indicating that the nonlinear effects associated with the separated flow are significant due to the high-amplitude flapping motion. This indicates that the TALF is still applicable for the separated flows around the airfoil at small Strouhal numbers. As argued by Sears (1976) it seems reasonable to use a vortex sheet as a model of a compressed viscous separated flow plus the airfoil. Therefore, the nonlinear effects are intrinsically incorporated into the TALF such that it could be applicable beyond the linear theories.

\subsection{Flapping rectangular morphing wing}

Dynamically stretching and retracting wingspan has been widely observed in the flight of birds and bats, and its effects on the aerodynamic performance particularly lift enhancement are intriguing. The overall lift enhancement of the morphing wing is affected by both the dynamic change of the lifting surface area and the altered flow structures by the dynamic morphing. A generic morphing flapping wing is proposed, as illustrated in Fig. 7, which characterizes the main features of the dynamically stretching and retracting wingspan. A rectangular flat-plate wing with a constant geometrical angle of attack (AoA) $\alpha$ heaves harmonically in a uniform freestream flow. Meanwhile, its wingspan stretches outward before reaching a certain position in the downstroke and retracts inward to the center line 
before reaching a certain position in the upstroke. The wing thickness is zero, and the wing chord remains constant. The flapping kinematics is described in a fixed laboratory coordinate system, as shown in Fig. 7.

The $x$-axis points downstream in the direction of the freestream flow, the $y$-axis is in the spanwise direction, and the $z$-axis is in vertical direction pointing upward. The flapping kinematics of the center of the wing is prescribed by $z_{w}=A \sin (2 \pi f t)$, where $z_{w}$ is the vertical position of the wing center, $A$ is the heaving amplitude, $f$ is the flapping frequency. The flapping Strouhal number is defined as $S t=2 f A / U_{\infty}$. The spanwise stretching and retracting motion of the wing has the same frequency as that of the flapping motion. The wingspan $L$ reaches the maximum in the downstroke and the minimum in the upstroke. The wing aspect ratio $(A R)$ is prescribed as a function of time, i.e., $A R(t)=L / c=A R_{0}(a-b \sin (2 \pi f t+\phi))$, where $A R_{0}=L_{0} / c$ is the characteristic aspect ratio, $L_{0}$ is the characteristic wingspan, $c$ is the constant chord, $a$ and $b$ are the coefficients that specify the stretching and retracting amplitude, and $\phi$ is the phase difference between the flapping and stretching/retracting motions. Figure 8 shows the time histories of the vertical displacement of the wing and the aspect ratio in the case of $S R=0.5$. The time-averaged $A R$ in a period is $\langle A R\rangle_{T}=a A R_{0}$. The span ratio, which is defined as a ratio between the minimum and maximum wingspans $\left(L_{\min }\right.$ and $L_{\max }$ ), is introduced to measure the magnitude of the stretching and retracting wingspan, i.e., $S R=L_{\min } / L_{\max }$. The span ratio depends on $a$ and $b$ for a given value of $A R_{0}$. There are the four kinematical parameters: the span ratio $S R$, the phase difference $\phi$, the flapping Strouhal number $S t=2 f A / U_{\infty}$, and the relative flapping magnitude $A^{*}=A / c$. The geometrical AoA $\alpha$ and the characteristic aspect ratio $A R_{0}$ are the other relevant parameters. The instantaneous wing area is $S(t)=L(t) c=c^{2} A R(t)$, and the time-averaged wing area in a period is $\langle S(t)\rangle_{T}=a c^{2} A R_{0}$.

For a dynamic morphing flapping wing with the time-dependent wing area, the lift coefficient in forward flight is defined as

$$
C l(t)=\frac{F_{z}(t)}{q_{\infty} S(t)},
$$


where $F_{z}$ is the lift acting on the wing, $q_{\infty}=0.5 \rho U_{\infty}^{2}$ is the dynamical pressure, $U_{\infty}$ is the freestream velocity (or forward flight velocity), and $S(t)$ is the instantaneous wing area. Theoretically, the effect of changing the wing area is removed in $C l(t)$ since $S(t)$ is used for normalization. To characterize the overall lift enhancement, the lift coefficient based on the time-averaged wing area $\langle S\rangle_{T}$ is introduced, i.e.,

$$
C l_{0}(t)=\frac{F_{z}(t)}{q_{\infty}\langle S\rangle_{T}} .
$$

To quantify the lift enhancement, the net increment of $\mathrm{Cl}_{0}(t)$ is defined as

$$
\Delta C l_{0}(t)=C l_{0}(t)-C l_{0, r e f}(t)
$$

where $\mathrm{Cl}_{0, \text {,ref }}(t)$ is the reference lift coefficient of a reference flapping wing with the fixed wingspan while the other parameters remain the same. This reference flapping wing has the fixed $A R$ that equals to the time-averaged $A R$ (i.e., $\langle A R\rangle_{T}=a A R_{0}$ ) of the corresponding dynamic morphing wing. Essentially, $\Delta C l_{0}(t)$ represents the lift increment generated by both the effect of changing the wing area and the effect of the flow structures altered by the dynamic morphing. In contrast, the instantaneous lift coefficient $C l(t)$ defined in Eq. (1) isolates the effect associated with the dynamic morphing by using the instantaneous wing area $S(t)$ for normalization. Similarly, to compare the wings with the dynamically changing wingspan and the fixed wingspan, the increment of $C l(t)$ is given by

$$
\Delta C l(t)=C l(t)-C l_{r e f}(t)
$$

where $\mathrm{Cl}_{r e f}(t)$ is the reference lift coefficient of the reference flapping wing with the fixed wingspan and the aspect ratio of $\langle A R\rangle_{T}$. In fact, $C l_{\text {ref }}(t)=C l_{0, r e f}(t)$ since the wing area is fixed for the reference wing. Here, $\Delta C l(t)$ mainly represents the lift increment generated by the effect of the morphing-altered flow structures. Furthermore, both $\Delta C l_{0}(t)$ and $\Delta C l(t)$ depend on the parameters $S R, \phi, S t, A^{*}$ and $\alpha$. The time-averaged quantity $\left\langle\Delta C l_{0}\right\rangle_{T}$ represents the overall lift enhancement, while $\langle\Delta C l\rangle_{T}$ 
describes the time-averaged lift enhancement caused by the effect of the morphing-altered flow structures when the effect of changing wing area is removed. In this paper, both $\left\langle\Delta C l_{0}\right\rangle_{T}$ and $\langle\Delta C l\rangle_{T}$ are used as the measures of the lift enhancement in the parametric space $\left(S R, \phi, S t, A^{*}, \alpha\right)$.

Numerical simulations of the low-Reynolds-number flows around the flapping rectangular morphing wing are conducted in the parametric space by using the immersed boundary method (Wang et al. 2014). Figure 9 shows the 3D flow structures viewed from the top for $\left(S R, \phi, S t, A^{*}, \alpha\right)=\left(0.5, \pi / 2,0.3,0.25,0^{\circ}\right)$ at the non-dimensional times $T^{*}=4.125, T^{*}=4.375, T^{*}=4.625, T^{*}=4.875$, and $T^{*}=5.125$, where the iso-surface of the second invariant $Q=3.0$ is shown and the colors indicate the spanwise vorticity.

We consider a typical case of the flapping wing with the stretching and retracting wingspan, where the kinematical and geometrical parameters are $\left(S R, \phi, S t, A^{*}, \alpha\right)=\left(0.5, \pi / 2,0.3,0.25,0^{\circ}\right)$ (referred to as the case of $S R=0.5$ ) for $A R_{0}=4$. For comparison, the corresponding wing with the fixed wingspan and $A R=3$ is considered, where $\left(S R, \phi, S t, A^{*}, \alpha\right)=\left(1.0, \pi / 2,0.3,0.25,0^{\circ}\right)$ (referred to as the case of $S R$ =1.0). Figure 10(a) shows the time histories of $C l_{0}(t)$ and $C l(t)$ in the cases of $S R=$ 0.5 and $S R=1.0$. Overall, both the lift coefficients $C l_{0}(t)$ and $C l(t)$ for $S R=0.5$ are larger than those for $S R=1.0$ during the most time of a flapping cycle. This observation indicates that the stretching and retracting wingspan motion can enhance the time-averaged lift. Further, since the effect of changing wing area is removed in $C l(t)$, the fact that $C l(t)$ for $S R=0.5$ is larger than that for $S R=1.0$ implies that the effect of the morphing-altered flow structures is responsible to the lift enhancement in addition to the effect of changing lifting surface area.

In the case of $S R=0.5$, the maximum of $C l_{0}(t)$ is reached when the wing is at about 1/3 of the downstroke. At the beginning of the upstroke, $C l_{0}(t)$ is at the minimum. The maximum magnitude of the positive $\mathrm{Cl}_{0}(t)$ generated in the downstroke is about two times larger than that of the negative $C l_{0}(t)$ in the upstroke. This asymmetric lift generation in the downstroke and upstroke results in $\left\langle C l_{0}\right\rangle_{T}>0 ;$ In the case of $S R=1.0$ where the 
wingspan remains constant during a flapping cycle, $\mathrm{Cl}_{0}(t)$ reaches the maximum when the wing moves about $1 / 3$ of the downstoke. The lift generation during the downstroke is similar to that in the $S R=0.5$ case, except that the maximum of $C l_{0}(t)$ is about $8 \%$ smaller. However, unlike the $S R=0.5$ case, the minimum of $C l_{0}(t)$ is reached when the wing is at about $1 / 3$ of the upstroke. The peak magnitude of the negative $C l_{0}(t)$ in the upstroke is equals to that of the positive $C l_{0}(t)$ in the downstroke. In contrast to the case of $S R=0.5$, this symmetric heaving kinematics without stretching and retracting wingspan in the downstroke and upstroke results in $\left\langle C l_{0}\right\rangle_{T}=O$.

Figure 10(b) shows the time histories of the lift coefficient increments $\Delta C l_{0}(t)$ and $\Delta C l(t)$ defined in Eqs. (28) and (29). The flapping wing generates a large positive lift increment $\left(\Delta C l_{0}>0\right)$ in about $2 / 3$ of the upstroke period for $S R=0.5$, which is mainly caused by both retracting the wingspan during the upstroke and altering the vortical structures associated with the spanwise motion. During the most time of the downstroke, $\Delta C l_{0}(t)$ remains positive although its value is relatively small. It is found that $\Delta C l(t))$ is positive in the most time of both the upstroke and downstroke and there are two peaks in this period. This fact indicates that the additional lift enhancement is achieved by the effect of the morphing-altered flow structures. Furthermore, it is found that $\left\langle\mathrm{Cl}_{0}\right\rangle_{T}=\langle\mathrm{Cl}\rangle_{T}=0$ for $S R$ $=1.0,\left\langle C l_{0}\right\rangle_{T}=0.81$ and $\langle C l\rangle_{T}=0.42$ for $S R=0.5$. The time-averaged lift coefficient $\langle C l\rangle_{T}=0.42$ for $S R=0.5$ is about half of $\left\langle C l_{0}\right\rangle_{T}=0.81$. This further confirms that the contribution to the lift enhancement by the morphing-altered flow structures is comparable to that generated by changing the lifting surface area. Figure 4 shows the time-averaged lift coefficient increments $\left\langle\Delta C l_{0}\right\rangle_{T}$ and $\langle\Delta C l\rangle_{T}$ as a function of $S R$ for $\phi=\pi / 2, S t=0.3$, $A^{*}=0.25$, and $\alpha=0^{\circ}$. The values of both $\left\langle\Delta C l_{0}\right\rangle_{T}$ and $\langle\Delta C l\rangle_{T}$ are positive while they monotonically decay as $S R$ increases, indicating that the lift enhancement is achieved for $0.25 \leq S R<1$ and the lift enhancement is larger for a smaller value of $S R$. Most importantly, it is found that that the lift enhancement $\left(\langle\Delta C l\rangle_{T}>0\right)$ of the morphing wing is the phenomenon not just in a special case but also in a large domain in the parametric space. 
The lift decomposition is used to further understand the relationship between the lift enhancement and the flow structures. Figure 12 shows the lift coefficient $C l_{\text {simp }}$ calculated by using the simple lift formula compared with $\mathrm{Cl}$ calculated based on the pressure and viscous stress fields on the wing. The contributions from the vortex lift and the acceleration term are denoted by $C l_{\text {vort }}$ and $C l_{a c c}$, respectively. As shown in Fig. 12, $C l_{\text {simp }}$ is in good agreement with $C l$. In the case of $S R=0.5$, the time-averaged lift coefficient calculated by using the simple lift formula is 0.43 , which agrees with 0.42 given by calculation based on the pressure and viscous stress fields on the wing surface. The relative error is about $2.4 \%$. For the case of $S R=1.0$, the positive lift and negative lift generated in the flapping are canceled out each other due to the symmetrical flapping motion such that the time-averaged lift coefficients $\langle C l\rangle_{T}$ and $\left\langle C l_{\text {simp }}\right\rangle_{T}$ are zero.

Figure 13 shows the contributions of the vortex lift term $\left(\Delta C l_{v o r t}\right)$ and local acceleration term $\left(\Delta C l_{a c c}\right)$ to the lift coefficient increment $\Delta C l$ in one period. It is indicated that the contribution of the vortex lift to $\Delta \mathrm{Cl}$ is positive in a full period particularly in the upstroke. This means that the vortex lift is enhanced by dynamically stretching and retracting wingspan in flapping flight. In this case, the local acceleration term has the negative contribution to $\triangle C l$ particularly in the upstroke. Furthermore, the contributions of the vortical structures to $\mathrm{Cl}$ in the upper and lower portions of the control volume divided by the flat-plate rectangular wing are evaluated in the cases of $S R=0.5$ and $S R=1.0$. Figure 14 shows the contributions of the vortex lift term to $\mathrm{Cl}$ in the upper and lower portions of the control volume in one period. In the average sense, the contributions of the vortex lift term in the upper and lower portions to $C l$ are positive and negative, respectively, in the cases of $S R=$ 0.5 and $S R=1.0$. During the upstroke, the contributions of the vortex force in both the upper and lower portions for $S R=0.5$ are larger than those for $S R=1.0$. The LEVs on the upper surface in the upstroke are significantly intensified by the spanwise vortex stretching, which contributes to the elevated lift in the case of $S R=0.5$. At the meantime, the shorter and more 3D LEVs on the lower surface have the smaller contribution to the negative lift. These differences lead to the higher positive peak in $\Delta C l(t)$ during the upstroke. During the downstroke, the contribution of the vortex force in the lower portion for $S R=0.5$ is still larger than that for $S R=1.0$. As a result, there is the smaller peak in $\Delta C l(t)$ during the downstroke. In summary, the vortex force associated with the vortical structures altered by dynamically changing wingspan significantly contributes the lift enhancement.

\subsection{Flapping bat wing}

Numerical simulations of the unsteady flow over a slow-flying bat are conducted to investigate the unsteady flow structures and aerodynamic lift generated by the bat flapping wings. The morphology of the bat wing is reconstructed based on the measurements of 
Watts et al. (2001), where the outline of the wing at its maximum wingspan is provided. The outline provided by Watts et al. (2001) is rescaled based on the relatively positions of the shoulder, wrist, wingtip, 5th digit and foot at the instant when the wingspan reaches the maximum according to the kinematics data of Wolf et al. (2010). The outline of the wing and the coordinate system is shown in Fig. 15. The kinematics of the wing in the present numerical model is reconstructed based on the measurements of Wolf et al. (2010), and the motions of the wingtip, wrist and 5th digit are prescribed while the shoulder and foot are fixed. The other points on the wing are interpolated from the five key points by using the bi-linear interpolation. The motions of the wingtip, wrist and 5th digit are fitted by using the Fourier series. It is noted that the bat studied by Watts et al. (2001) for the wing morphology is the grey-headed flying fox (Pteropus poliocephalus), while the bat studied by Wolf et al. (2010) for the wing kinematics is the Pallas long-tongued bats (Glossophaga soricina). In this sense, the wing geometric and kinematic model in this work is a combination of the two different bat species which serves as a generic bat model. The Reynolds number based on the wing chord $c$ and the freestream velocity $U_{\infty}$ is $R e=U_{\infty} c / v=1000$ and the Strouhal number is $S t=f_{c} / U_{\infty}=1.36$, where $f$ is the flapping frequency.

Figure 16 shows the top views of the vortical structures around the flapping bat wings at four different phases of a flapping cycle (the start of downstroke, middle of downstroke, start of upstroke, and middle of upstroke), where the vortical structures are identified using the $\lambda_{2}$-criterion (Jeong \& Hussian 1995) and colored by the streamwise vorticity. The distinct features are the leading-edge vortices (LEVs) generated in the downstroke that are responsible to the vortex lift generation. In contrast, there is no strong and coherent LEV generated in the upstroke. This observation is consistent with the previous reports that the stable LEVs correspond to high lift generation (Muijres et al. 2008). Figure 17 shows the spanwise vorticity distributions in three spanwise slices when the bat wingspan reaches the maximum in the downstroke. The flow fields in the left column are from the PIV measurements of a Pallas long-tongued bat at $1.5 \mathrm{~m} / \mathrm{s}$ by Muijures et al. (2008). The flow fields in the right column are from the DNS in the present model at a speed of $1 \mathrm{~m} / \mathrm{s}$. It is found the vorticity distributions in the three spanwise slices obtained from the DNS are very similar to those obtained in the PIV measurements by Muijures et al. (2008). The vortex shedding from the leading edge on the upper surface can be observed in both the DNS and measurements, which considerably contribute the vortex lift generation. The wake structures are also similar in the DNS and measurements.

The lift coefficient $C l(t)=L(t) / q_{\infty} S_{\max }$ is calculated in one flapping period, where $L$ is the lift, $q_{\infty}=\rho U_{\infty}^{2} / 2$ is the dynamic pressure, $S_{\max }$ is the maximum orthographically projected wing area, and $U_{\infty}$ is the forward flight velocity. The drag coefficient is defined similarly. Figure 18 shows the time histories of the lift coefficients of 
the flying bat in one period. The time history of $\mathrm{Cl}$ is similar to that for a fruit bat at a higher Reynolds number (Viswanath et al. 2014). The time-averaged lift coefficient in one flapping period is $\langle\mathrm{Cl}\rangle_{T}=12.9$, where $\langle\bullet\rangle_{T}$ is a time-averaging operator. To elucidate the relationship between the lift generation and the vortical structures, the lift decomposition is applied. The coefficients of the vortex lift and the lift associated with the fluid acceleration are defined as $C l_{v o r}=L_{v o r} / q_{\infty} S_{\max }$ and $C l_{a c c}=L_{a c c} / q_{\infty} S_{\max }$, respectively. The time histories of $C l_{v o r}$ and $C l_{a c c}$ in one flapping period are shown in Fig. 18. Interestingly, it is found that the vortex lift coefficient $C l_{v o r}$ is positive in both the downstroke and upstroke, indicating that the LEVs can still contribute lift generation even when they are detached from the wing in the upstroke. The time-averaged vortex lift coefficient of the flying bat is $\left\langle C l_{v o r}\right\rangle_{T}=9.2$ that is about $71 \%$ of the total time-averaged lift coefficient. As observed in Fig. 18, $\mathrm{Cl}_{a c c}$ is positive in the downstroke and negative in most of the upstroke, and it has also the positive contribution to the time-averaged lift. The time-averaged value is $\left\langle C l_{a c c}\right\rangle_{T}=3.7$ that is about $29 \%$ of the total time-averaged lift coefficient.

\section{Conclusions}

The complex flows around flapping wings at low Reynolds numbers are highly unsteady and separated, which contain the leading-edge vortices, tip vortices and wake vortices. The relationship between lift generation and complex flow structures is critical to understand the physical mechanisms of flapping flight. The simple lift formula is given for a wing in a rectangular control volume in the viscous-flow framework, and it has a lucid form with the two leading-order terms: the Lamb vector integral for the vortex force and the acceleration term of fluid for the unsteady inertial effect. Therefore, the lift problem in flapping forward flight at low Reynolds numbers can be addressed based on the application of the simple lift decomposition. Due to its simplicity, the simple lift formula is particularly useful for estimation and analysis of the unsteady lift from velocity data obtained in measurements and computations. The Kutta-Joukowski theorem is a reduced case from the simple lift formula for a lumped vorticity patch in a steady-state flow. Further, the physical foundations of the unsteady thin airfoil theory are explored by using the simple lift formula in the viscous-flow framework. The thin-airfoil lift formula is derived that could be applicable even when the flow around an airfoil is moderately separated, and the classical Kárman-Sears lift formula is recovered as a reduced case. The simple lift formula is further validated through direct numerical simulation of the flows on the flapping flat-plate airfoil, the flapping rectangular morphing wing and the flapping bat wing. The lift-generating/enhancing mechanisms in these cases particularly the roles of the leading-edge vortices are revealed based on the simple lift decomposition. 


\section{Acknowledgements}

This work was supported by the National Natural Science Foundation of China under Project Nos. 10872201, 11232011, 11302238 and 11372331, and the National Basic Research Program of China (973 Program) under Project No. 2013CB834100 (Nonlinear science). Tianshu Liu would like to acknowledge the hospitality received at LNM during his visit where he accomplished this work. The simulations were performed on TianHe-1. We would like to acknowledge the support from the National Supercomputer Center in Tianjin.

\section{References:}

Ansari, S. A., Zbikowski, R., and Knowles, K. "Aerodynamic Modelling of Insect-Like Flapping Flight for Micro Air Vehicles," Progress in Aerospace Sciences Vol. 42, No. 2, 2006, pp. 129-172.

Chang, C.-C. "Potential Flow and Forces for Incompressible Viscous Flow," Proceedings of the Royal Society of London. Series A: Mathematical and Physical Sciences Vol. 437, No. 1901, 1992, pp. 517-525.

Chang, W., Giraldo, F., and Perot, B. "Analysis of an Exact Fractional Step Method," Journal of Computational Physics Vol. 180, No. 1, 2002, pp. 183-199.

Glauert, H., "The Elements of Aerofoil and Airscrew Theory (Second Edition)," Cambridge University Press, 1983, Chapter VII.

Howe, M. S. "On Unsteady Surface Forces, and Sound Produced by the Normal Chopping of a Rectilinear Vortex," Journal of Fluid Mechanics Vol. 206, 1989, pp. 131-153.

Hedenstrom, A., Johansson, L. C., Wolf, M., von Busse, R., Winter, Y., and Spedding, G. R. "Bat Flight Generates Complex Aerodynamic Tracks," Science Vol. 316, No. 5826, 2007, pp. 894-897.

Hubel, T. Y., Hristov, N. I., Swartz, S. M., and Breuer, K. S. "Time-Resolved Wake Structure and Kinematics of Bat Flight," Experiments in Fluids Vol. 46, No. 5, 2009, pp. 933-943.

Hubel, T. Y., Hristov, N. I., Swartz, S. M. , Breuer, K. S. "Time-resolved wake structure and kinematics of bat flight," Experiments in Fluids 46, 2009, pp. 933-943.

Jeong, J., Hussain, F., "On the Identification of a Vortex," Journal of Fluid Mechanics 285, 1995, pp. 69-94.

von Káman, T. and Sears, W. R., "Airfoil Theory for Non-Uniform Motion," Journal of Aeronautical Sciences, Vol. No. 10, pp. 379-390, 1938.

von Karman, T. and Burgers, J., "General Aerodynamic Theory -- Perfect Fluids," in Aerodynamic Theory, F. Durand, Editor (Dover publications, 1963).

Lee, J. J., Hsieh, C. T., Chang, C. C., and Chu, C. C. "Vorticity Forces on an Impulsively Started Finite Plate," Journal of Fluid Mechanics Vol. 694, 2012, pp. 464-492.

Liu, T. S., Wang, S. Z., Zhang, X., He, G. W., and " Unsteady Thin Airfoil Theory Revisited: Application of a Simple Lift Formula," AIAA J. (published online) 2014.

Muijres, F. T., Johansson, L. C., Barfield, R., Wolf, M., Spedding, G. R. and Hedenstrom. A., "Leading-Edge Vortex Improves Lift in Slow-Flying Bats," Science 319, 2008, pp. 1250-1253.

Noca, F., Shiels, D., and Jeon, D. "A Comparison of Methods for Evaluating Time-Dependent Fluid Dynamic Forces on Bodies, Using Only Velocity Fields and Their Derivatives," 
Journal of Fluids and Structures Vol. 13, No. 5, 1999, pp. 551-578.

Platzer, M. F., Jones, K. D., Young, J. and S. Lai, J. C. "Flapping Wing Aerodynamics:

Progress and Challenges," AIAA Journal Vol. 46, No. 9, 2008, pp. 2136-2149.

Quartapelle, L., and Napolitano, M. "Force and Moment in Incompressible Flows," AIAA Journal Vol. 21, No. 6, 1983, pp. 911-912.

Rayner, J. M. V. "Vortex Theory of Animal Flight 1. Vortex Wake of a Hovering Animal," Journal of Fluid Mechanics Vol. 91, No. APR, 1979, pp. 697-730.

Rayner, J. M. V. "Vortex Theory of Animal Flight 2. Forward Flight of Birds," Journal of Fluid Mechanics Vol. 91, No. APR, 1979, pp. 731-763.

Saffman, P. G. Vortex Dynamics. Cambridge: Cambridge University Press, 1992.

Sane, S. P. "The Aerodynamics of Insect Flight," Journal of Experimental Biology Vol. 206, No. 23, 2003, pp. 4191-4208.

Sears, W. R., "Unsteady Notion of Airfoil with Boundary-Layer Separation," AIAA J., Vol. 14, No. 2, 1976, pp. 216-220.

Shyy, W., Lian, Y., Tang, J., Viieru, D., and Liu, H. Aerodynamics of Low Reynolds Number Flyers. Cambridge: Cambridge University Press, 2008.

Spedding, G. R., Rosen, M., and Hedenstrom, A. "A Family of Vortex Wakes Generated by a Thrush Nightingale in Free Flight in a Wind Tunnel over Its Entire Natural Range of Flight Speeds," Journal of Experimental Biology Vol. 206, No. 14, 2003, pp. 2313-2344.

Viswanath, K., Nagendra, K., Cotter, J., Frauenthal, M., Tafti. D., "Straight-line Climbing Flight Aerodynamics of a Fruit Bat. Physics of Fluids 26, 021901 (2014).

Wang, S. Z., Zhang, X., He, G. W., and Liu, T. S. "A Lift Formula Applied to Low-Reynolds-Number Unsteady Flows," Physics of Fluids, Vol. 25, 2013, p. 093605.

Wang, S. Z., and Zhang, X. "An Immersed Boundary Method Based on Discrete Stream Function Formulation for Two- and Three-Dimensional Incompressible Flows," Journal of Computational Physics Vol. 230, No. 9, 2011, pp. 3479-3499.

Wang, S., He, G., Zhang, X. and Liu, T., "Lift Enhancement by Dynamically Changing Wingspan in Forward Flapping Flight," Physics of Fluids, Vol. 26, 2014a, p. 061903.

Wang, S., Zhang, X, He, G., and Liu, T., "Evaluation of lift formulas applied to low Reynolds number flows," AIAA Journal (published online), 2014b.

Watts, P., Mitchell, E. J., and Swartz, S. M. "A Computational Model for Estimating the Mechanics of Horizontal Flapping Flight in Bats: Model Description and Validation," Journal of Experimental Biology Vol. 204, No. 16, 2001, pp. 2873-2898.

Wolf, M., Johansson, L. C., von Busse, R., Winter, Y., Hedenstrom. A., "Kinematics of flight and the relationship to the vortex wake of a Pallas' long tongued bat (Glossophaga soricina)," Journal of Experimental Biology 213, 2010, 2142-2153.

Wu, J.-Z., Pan, Z.-L., and Lu, X.-Y. "Unsteady Fluid-Dynamic Force Solely in Terms of Control-Surface Integral," Physics of Fluids Vol. 17, No. 9, 2005, p. 098102.

Wu, J. Z., Ma, H. Y., and Zhou, M. D. Vorticity and Vortex Dynamics: Springer, 2006. 
Table 1. Kinematical Parameters of a Flapping Flat-Plate Airfoil

\begin{tabular}{c|c|c|c|c|c}
\hline Case & $k=\pi f c / U$ & $A / c$ & $\alpha_{0}$ & $\alpha_{m}$ & $z_{c 0} / c$ \\
\hline$A$ & $0.06 \pi$ & 0.025 & $1^{\circ}$ & $3^{\circ}$ & 0 \\
\hline$B$ & $0.6 \pi$ & 0.025 & $1^{\circ}$ & $3^{\circ}$ & 0 \\
\hline$C$ & $0.06 \pi$ & 0.25 & $10^{\circ}$ & $30^{\circ}$ & 0 \\
\hline$D$ & $0.6 \pi$ & 0.25 & $10^{\circ}$ & $30^{\circ}$ & 0 \\
\hline
\end{tabular}

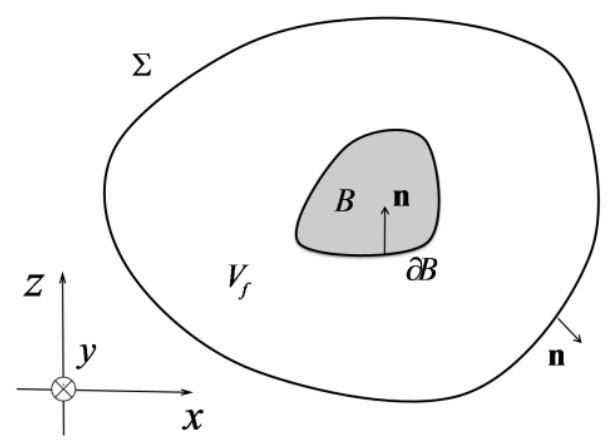

Figure 1. Schematic of a control surface around a body.

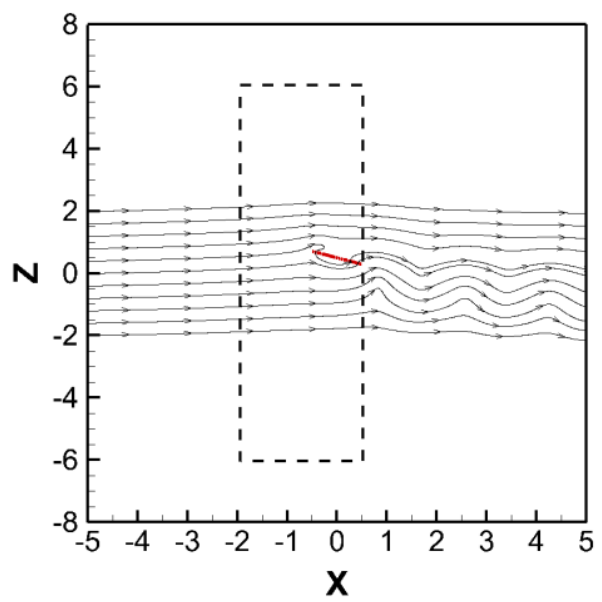

Figure 2. Schematic of a rectangular control volume.

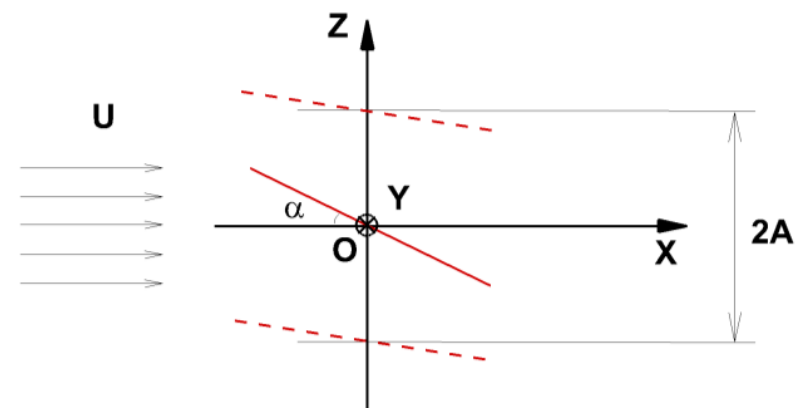

Figure 3. The flapping flat-plate airfoil and the coordinate system in DNS. 


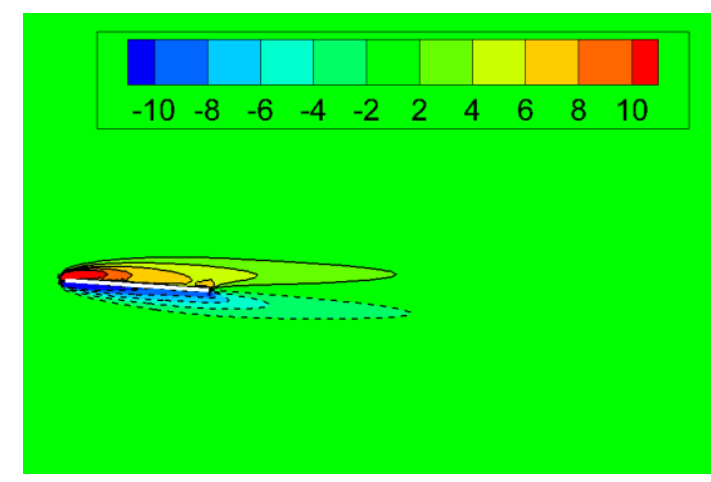

(a)

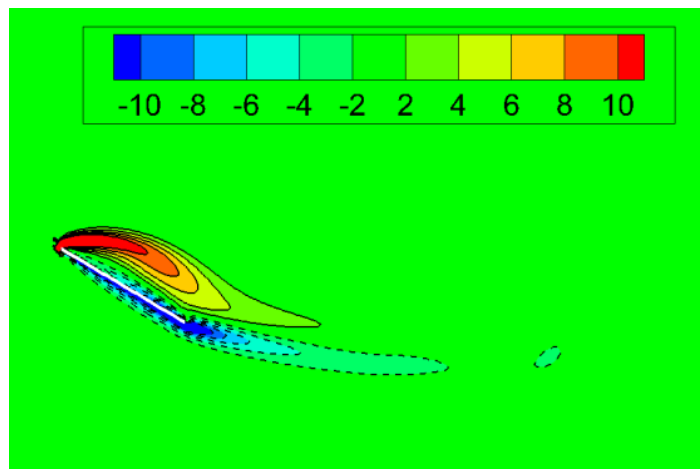

(c)

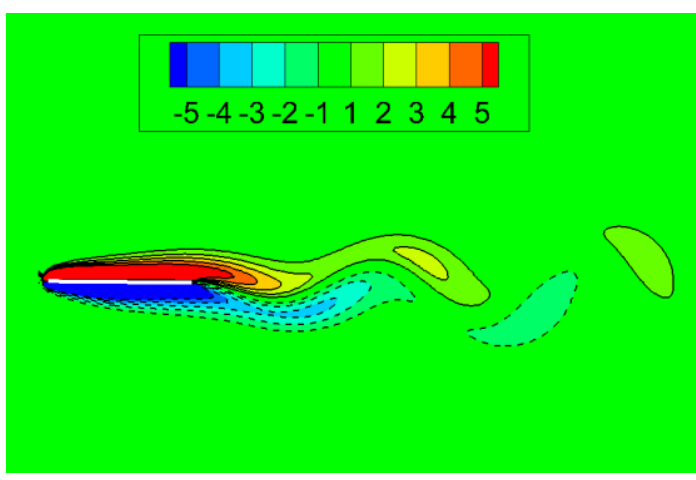

(b)

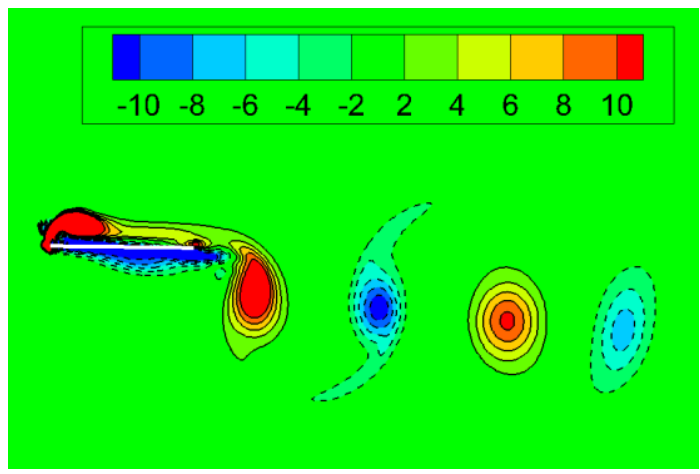

(d)

Figure 4. The vorticity fields around the plate when the lift reaches the maximum in (a) Case A, (b) Case B, (c) Case C, and (d) Case D.

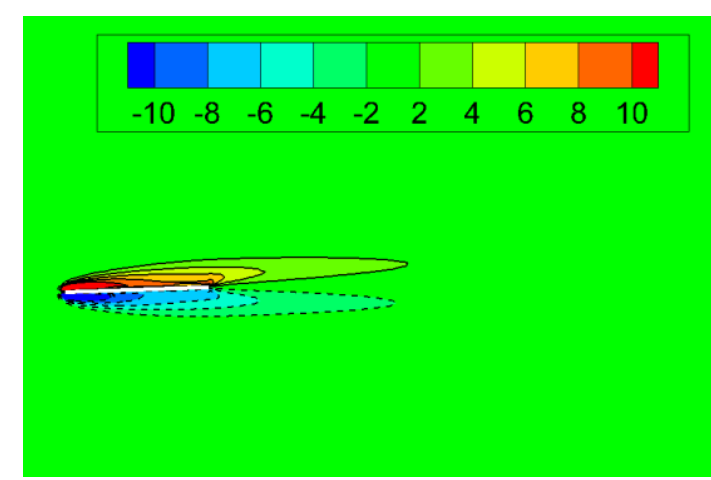

(a)

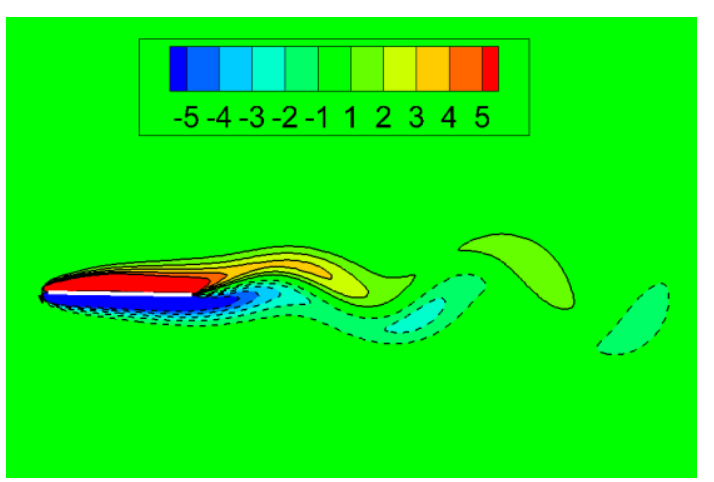

(b)
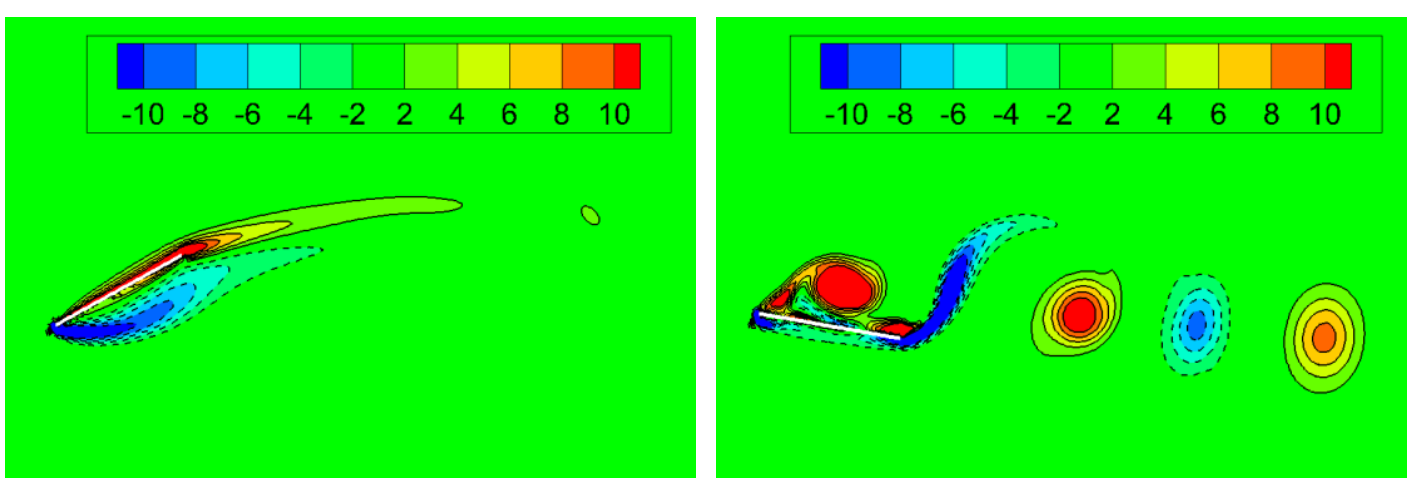

(c)

(d)

Figure 5. The vorticity fields around the plate when the lift reaches the minimum in (a) Case A, (b) Case B, (c) Case C, and (d) Case D. 


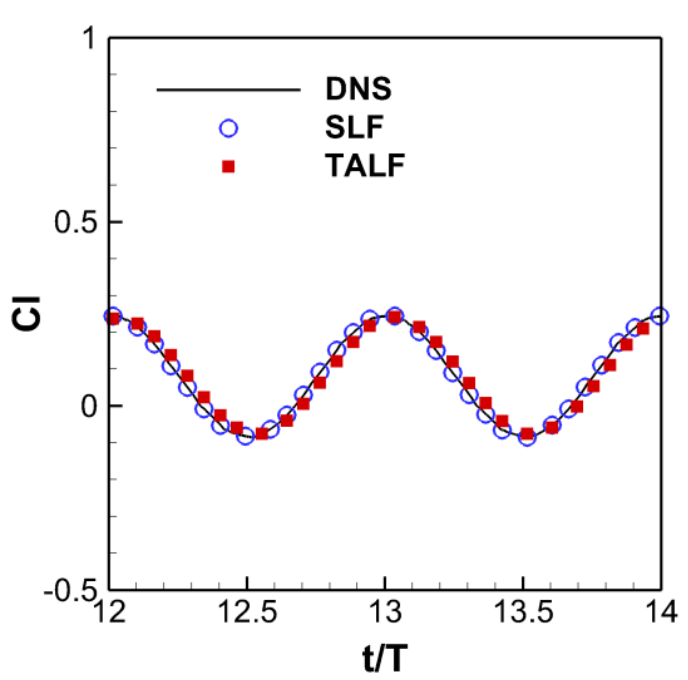

(a)

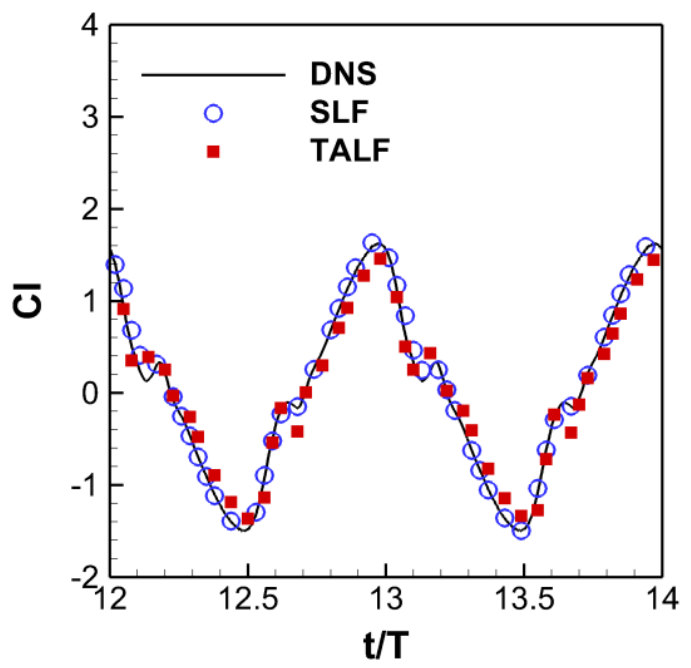

(c)

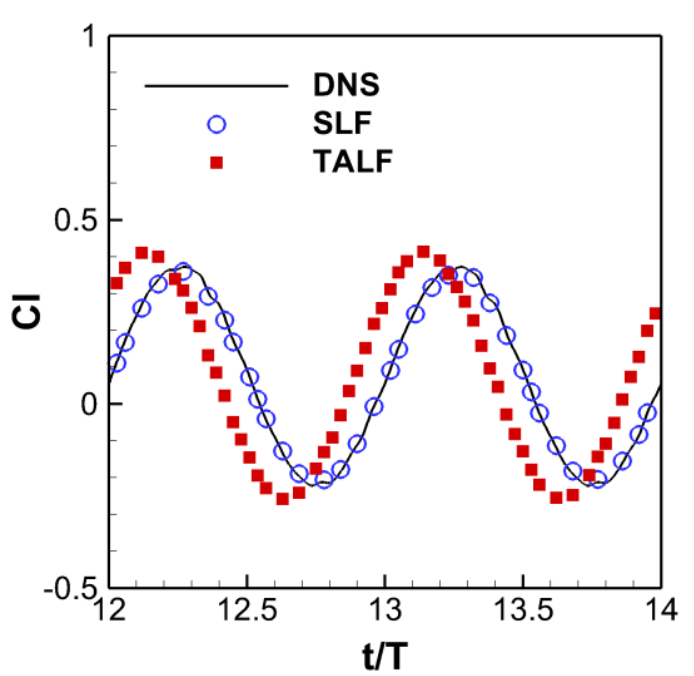

(b)

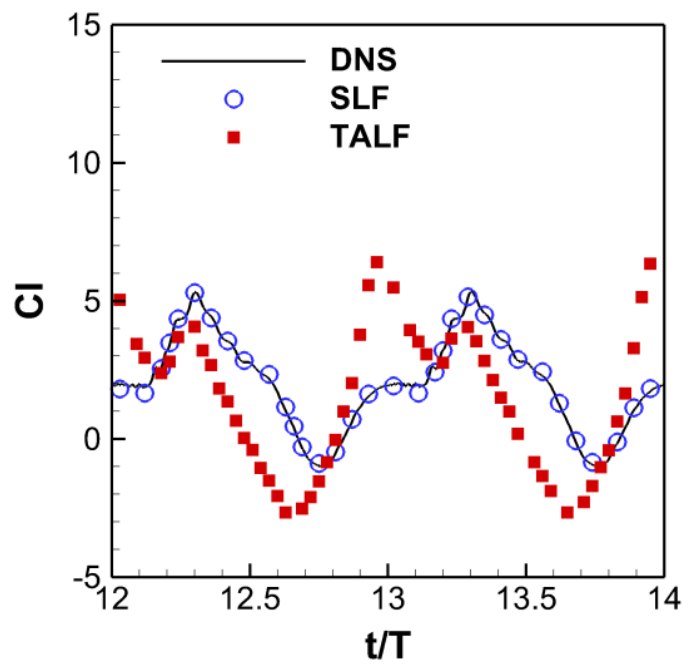

(d)

Figure 6. The lift coefficients given by the thin-airfoil lift formula (TALF), the simple lift formula (SLF) and DNS in (a) Case A, (b) Case B, (c) Case C, and (d) Case D. 
(a)

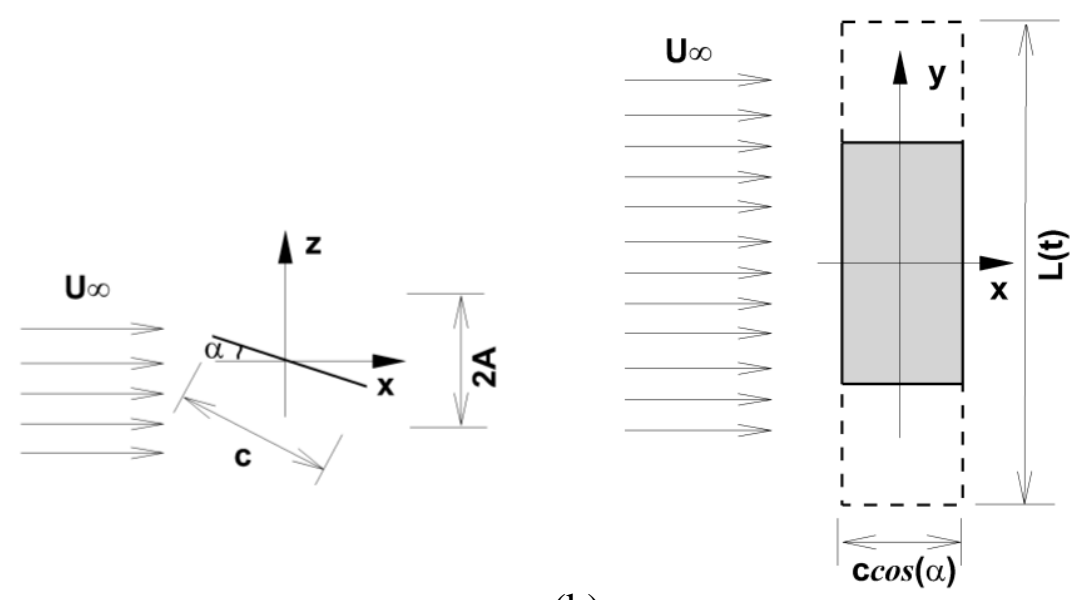

(b)

Figure 7. Schematic of the computational model: (a) side view and (b) top view.

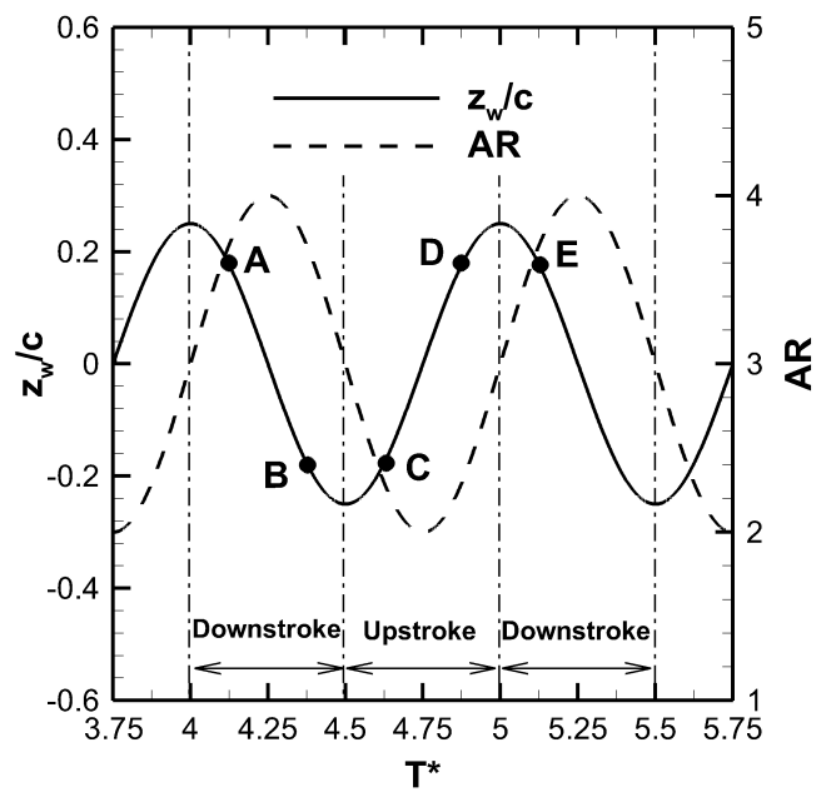

Figure 8. The time histories of the vertical displacement of the wing and the aspect ratio in the case of $S R=0.5$. The solid circles A, B, C, D and E denote five key moments $T^{*}=4.125,4.375,4.625,4.875$ and 5.125 , respectively. 
(a)
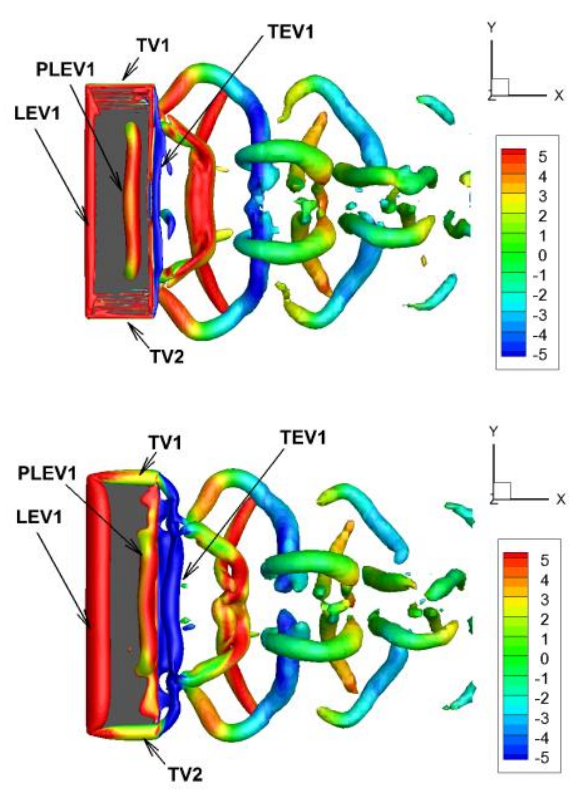

(b)

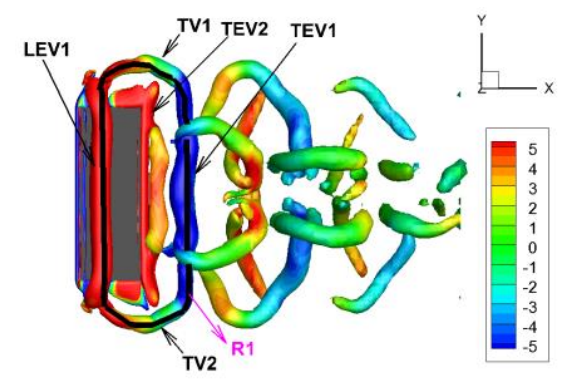

(d)

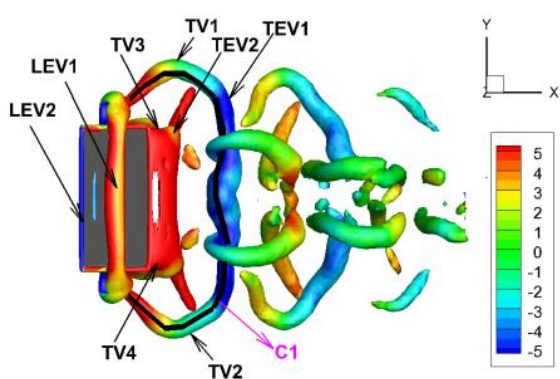

(e)

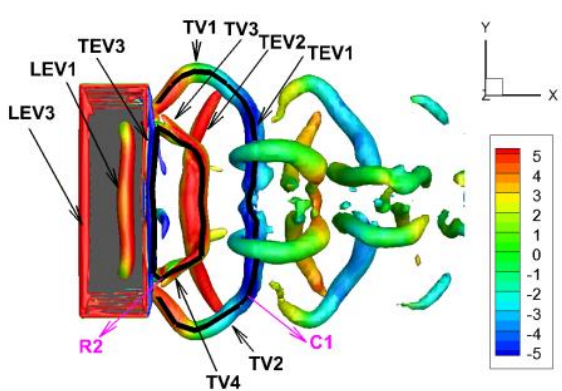

(c)

Figure 9. The three-dimensional flow structures viewed from the top for $\left(S R, \phi, S t, A^{*}, \alpha\right)=\left(0.5, \pi / 2,0.3,0.25,0^{\circ}\right)$ at different time (a) $T^{*}=4.125$, (b) $T^{*}=4.375$, (c) $T^{*}=4.625$, (d) $T^{*}=4.875$, and (e) $T^{*}=5.125$. The iso-surface of $Q=3.0$ is shown, where the colors indicate the spanwise vorticity.

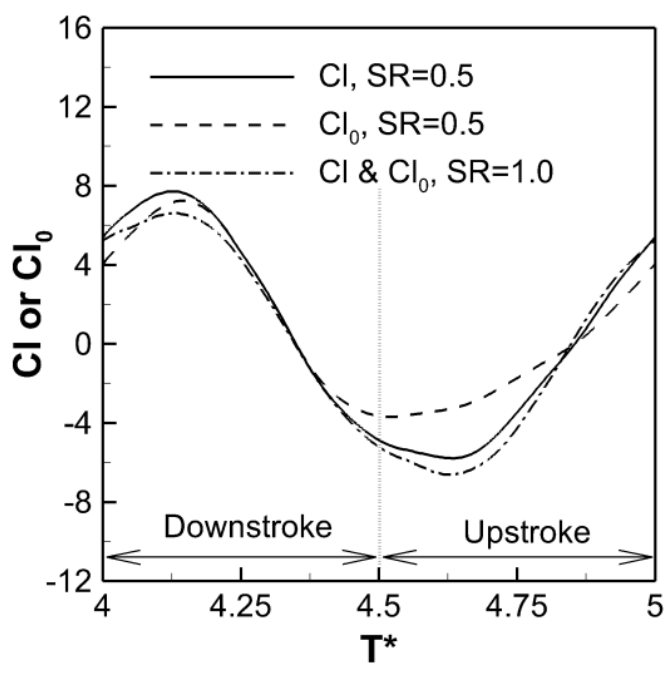

(a)

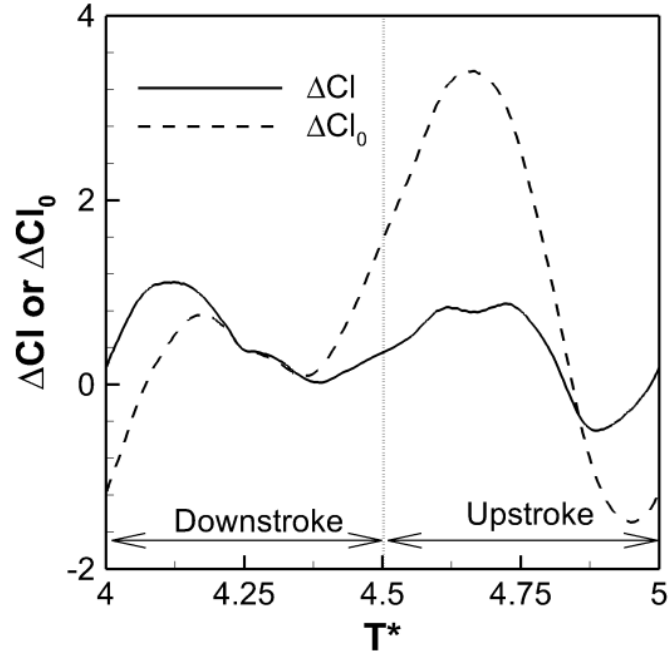

(b)

Figure 10. The time histories of (a) $C l$ and $C l_{0}$, and (b) $\Delta C l$ and $\Delta C l_{0}$ in one period in the cases of $S R=0.5$ and $S R=1.0$ for $\phi=\pi / 2, S t=0.3, A^{*}=0.25$, and $\alpha=0^{\circ}$. 


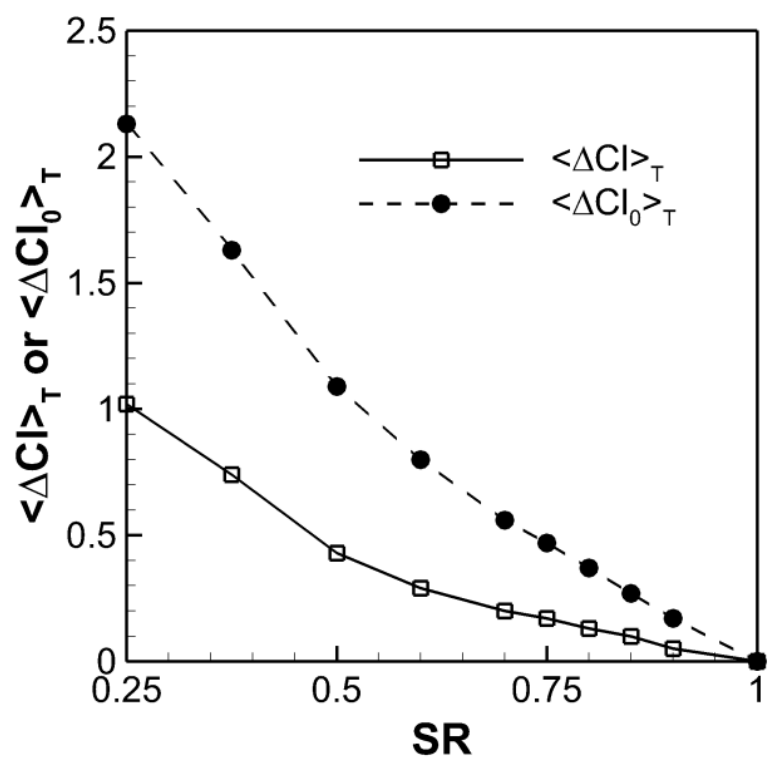

Figure 11. The time-averaged increments of the lift coefficient $\left\langle\Delta C l_{0}\right\rangle_{T}$ and $\langle\Delta C l\rangle_{T}$ as a function of the span ratio $S R$ for $\phi=\pi / 2, S t=0.3$, and $A^{*}=0.25$, and $\alpha=0^{\circ}$.

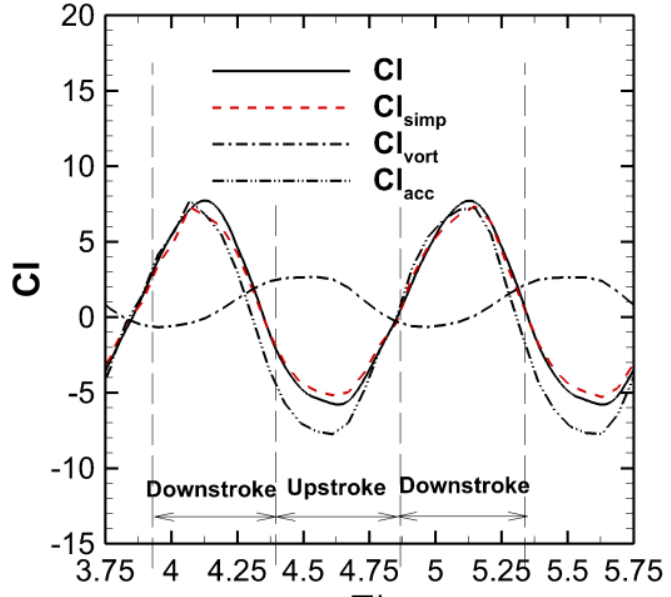

(a)

(b)

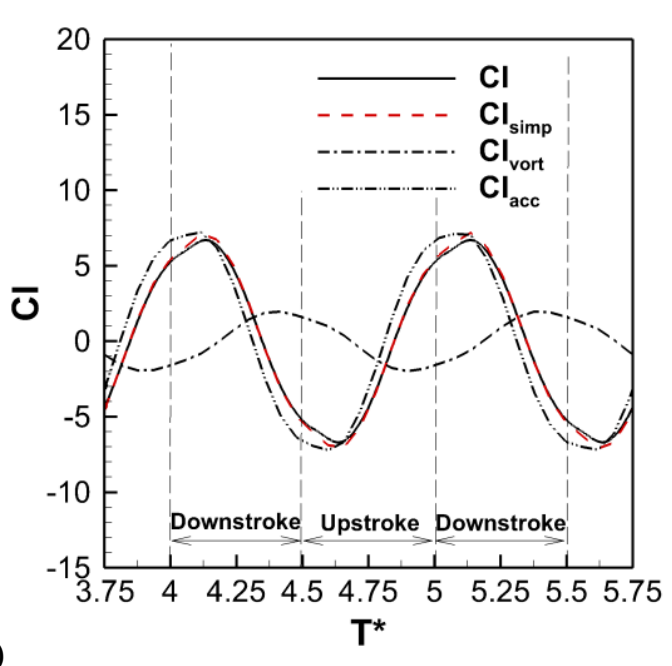

(b)

$\phi=\pi / 2, S t=0.3, A^{*}=0.25$ and $\alpha=0^{\circ}$. 


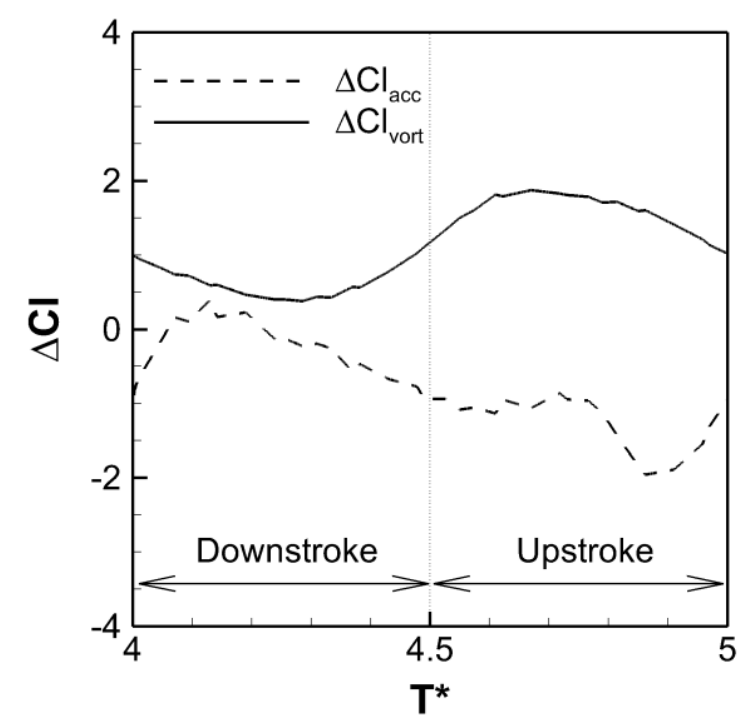

Figure 13. The contributions of the Lamb vector term (the vortex force) and local acceleration term to the increment of the lift coefficient $\Delta C l$ in one period for $\phi=\pi / 2, S t=0.3$, $A^{*}=0.25$ and $\alpha=0^{\circ}$.

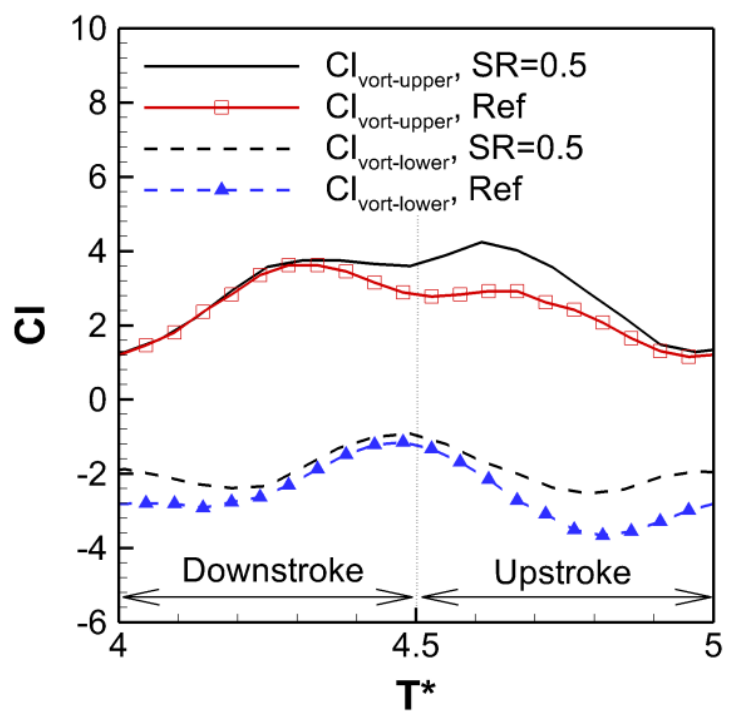

Figure 14. The contributions of the Lamb vector term to the lift coefficient $C l$ in the upper and lower portions of the control volume for $\phi=\pi / 2, S t=0.3, A^{*}=0.25$ and $\alpha=0^{\circ}$.

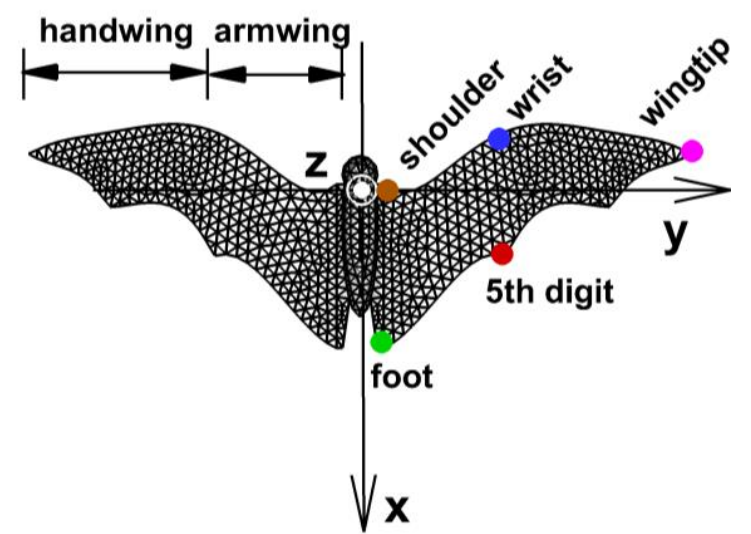

Figure 15. The rescaled outline of the bat wing based on the measurements of Watts et al. (2001). 


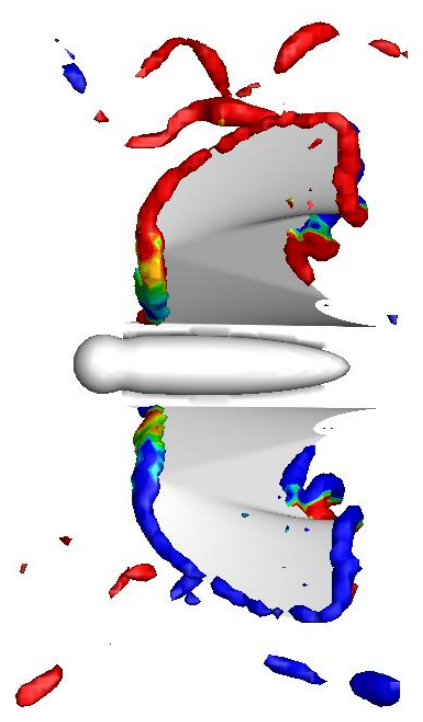

(a)

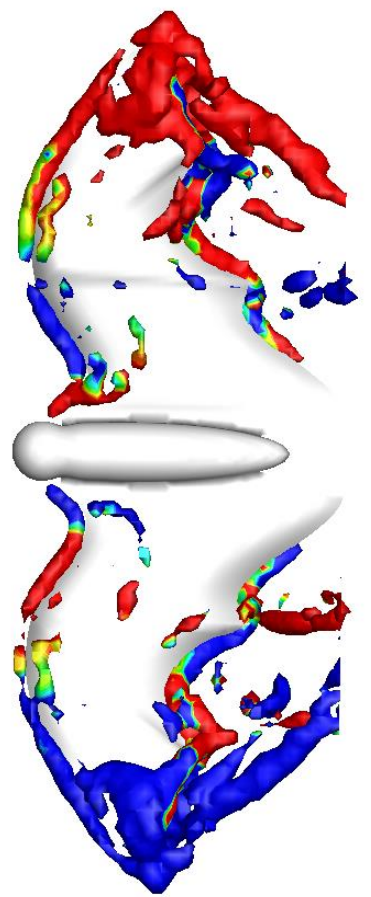

(b)

Figure 16. The vortical structures around the slow-flying bat at (a) start of downstroke, (b) middle of downstroke, (c) start of upstroke, (d) middle of upstroke. The vortical structures are identified by using the $\lambda_{2}$-criterion $\left(\lambda_{2}=-150\right)$. The color shows the streamwise vorticity.

(c)

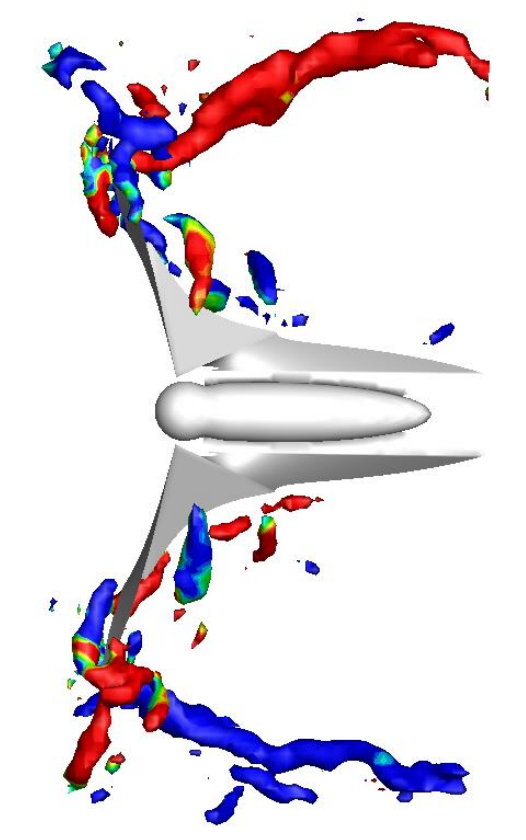

(d)

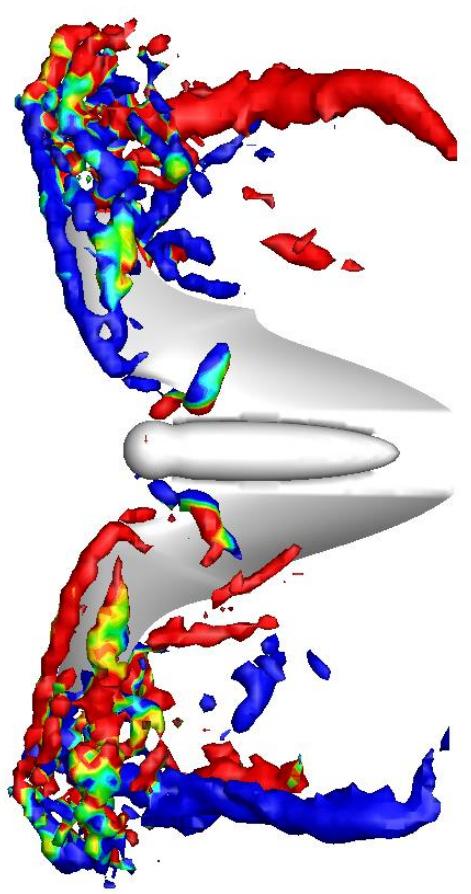

)

flying bat at (a) start of downstroke, (b)
50 ). The color shows the streamwise 

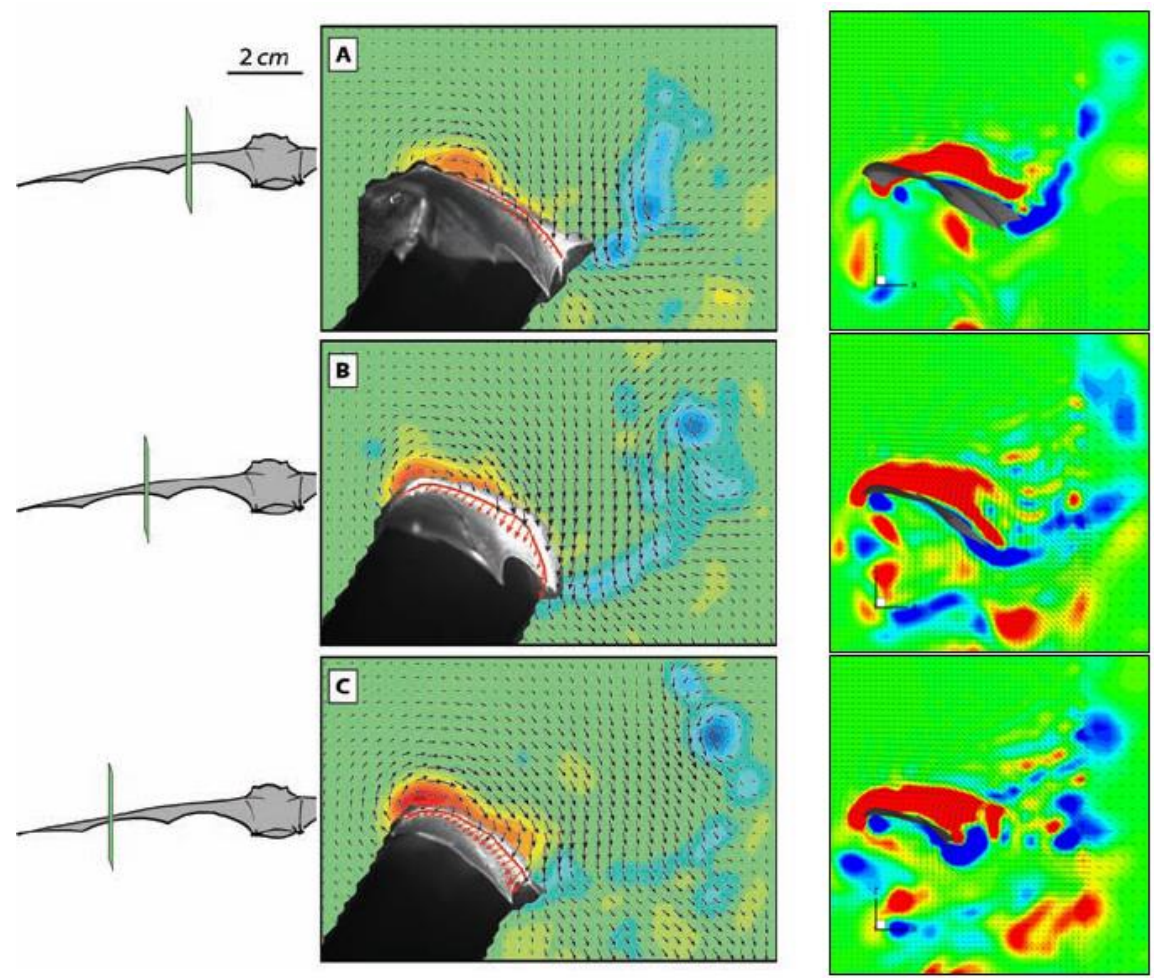

Figure 17. The spanwise vorticity distributions in three spanwise slices when the bat wingspan reaches the maximum in the downstroke. The flow fields in the left column are obtained from the PIV measurements of a Pallas long-tongued bat at $1.5 \mathrm{~m} / \mathrm{s}$ by Muijures et al. (2008). The flow fields in the right column are obtained from the DNS in the present model at a speed of $1 \mathrm{~m} / \mathrm{s}$.

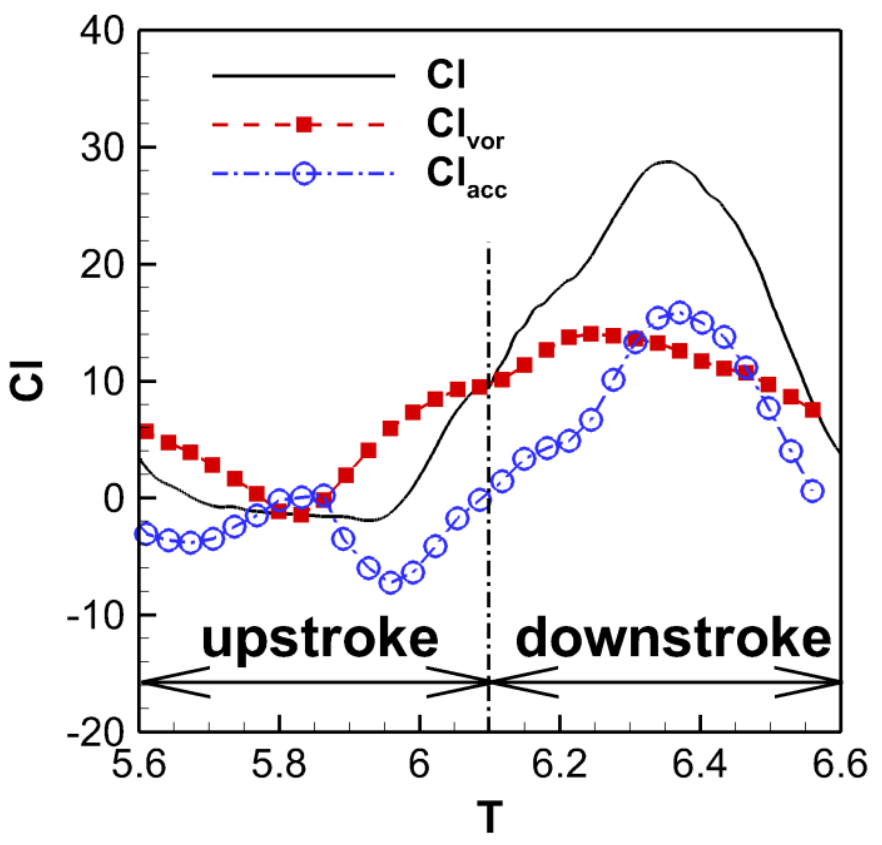

Figure 18. Time histories of the lift coefficients of the flying bat in one period. 